\title{
Archaeal TFE $\alpha / \beta$ is a hybrid of TFIIE and the RNA polymerase III subcomplex hRPC62/39
}

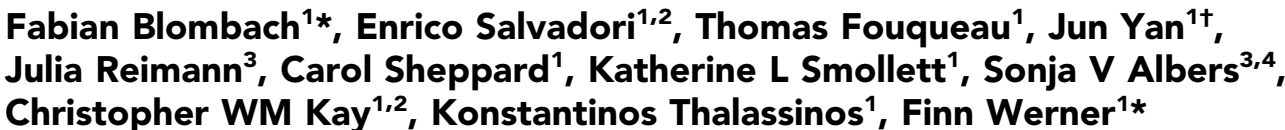

${ }^{1}$ Institute for Structural and Molecular Biology, Division of Biosciences, University College London, London, United Kingdom; ${ }^{2}$ London Centre for Nanotechnology, University College London, London, United Kingdom; ${ }^{3}$ Molecular Biology of Archaea Group, Max Planck Institute for Terrestrial Microbiology, Marburg, Germany; ${ }^{4}$ Microbiology, University of Freiburg, Freiburg, Germany

Abstract Transcription initiation of archaeal RNA polymerase (RNAP) and eukaryotic RNAPII is assisted by conserved basal transcription factors. The eukaryotic transcription factor TFIIE consists of $\alpha$ and $\beta$ subunits. Here we have identified and characterised the function of the TFIIE $\beta$ homologue in archaea that on the primary sequence level is related to the RNAPIII subunit hRPC39. Both archaeal TFE $\beta$ and hRPC39 harbour a cubane 4Fe-4S cluster, which is crucial for heterodimerization of $\mathrm{TFE} \alpha / \beta$ and its engagement with the RNAP clamp. TFE $\alpha / \beta$ stabilises the preinitiation complex, enhances DNA melting, and stimulates abortive and productive transcription. These activities are strictly dependent on the $\beta$ subunit and the promoter sequence. Our results suggest that archaeal $\mathrm{TFE} \alpha / \beta$ is likely to represent the evolutionary ancestor of TFIIE-like factors in extant eukaryotes. DOI: 10.7554/eLife.08378.001

f.blombach@ucl.ac.uk (FB);

f.werner@ucl.ac.uk (FW)

Present address: ${ }^{\dagger}$ Department of Chemistry, University of Oxford, Oxford, United Kingdom

Competing interests: The authors declare that no competing interests exist.

Funding: See page 20

Received: 28 April 2015

Accepted: 11 June 2015

Published: 12 June 2015

Reviewing editor: Patrick Cramer, Max Planck Institute for Biophysical Chemistry, Germany

C) Copyright Blombach et al. This article is distributed under the terms of the Creative Commons Attribution License, which permits unrestricted use and redistribution provided that the original author and source are credited.

\section{Introduction}

The conserved core of the archaeal and eukaryotic transcription machineries encompasses a highly complex multisubunit RNAP as well as evolutionary conserved transcription factors that govern its activities through the transcription cycle. The minimal requirements for promoter-directed and start site-specific transcription are identical for the RNAPII system in eukaryotes (Parvin and Sharp, 1993) and the RNAP of archaea (Hausner et al., 1996; Qureshi et al., 1997; Werner and Weinzierl, 2002). Two basal transcription factors, TBP (TATA-binding protein) and TFB (TFIIB in eukaryotes), are necessary and sufficient to initiate transcription in archaea in vitro. TBP and TFB facilitate promoter recognition and the recruitment of RNAP (Werner and Grohmann, 2011). In archaea as well as eukaryotes a third factor TFE (TFIIE) enhances the next step in initiation, the transition of the closed to the open complex (Holstege et al., 1995, 1996; Werner and Weinzierl, 2005). Eukaryotic TFIIE is a heterodimer composed of subunits TFIIE $\alpha$ and TFIIE $\beta$ in humans (Tfa1 and 2 in yeast). Archaeal TFE (hereafter referred to as TFE $\alpha$ ) is monomeric and homologous to TFIIE $\alpha$, but lacks its C-terminal acidic domain (Bell et al., 2001; Hanzelka et al., 2001). To date no archaeal homologue of TFIIE $\beta$ has been identified. During open complex formation the DNA strands are separated (melted) and the template strand is loaded into the active centre of RNAP. Similar to TFIIE, TFE $\alpha$ facilitates open complex formation by directly interacting with the non-template DNA strand (NTS), and via an allosteric mechanism that is likely to involve structural changes in the RNAP clamp and stalk (Grohmann et al., 2011). While in archaea the closed-to-open complex transition occurs spontaneously (Werner and Weinzierl, 2002), on most eukaryotic RNAPII promoters it is dependent on the translocase activity of 
eLife digest Life on Earth is often categorized into three domains: the eukaryotes (which include plants, animals and fungi), the bacteria and a group of unusual, single-celled microorganisms called the archaea. But several recent discoveries suggest that the origin of the eukaryotes lies within the archaeal domain. The genetic material of all of these living organisms is made up of DNA, and genes within DNA contain the instructions to make other biological molecules. Making these molecules involves first copying these instructions into a molecule of RNA via a process called transcription.

All three domains of life use enzymes called RNA polymerases (RNAPs) for transcription, and all RNAPs are thought to have originated from a common ancestor. Archaea and bacteria have a single type of RNAP, whereas all eukaryotes have at least four different kinds of RNAP. The RNAPs found in archaea share many common features with their eukaryotic counterparts. In both cases, the RNAPs do not work alone. Instead, a class of proteins known as transcription factors assist in the first step of the transcription process. One of the eukarotyic RNAPs, termed RNAP II, works with a transcription factor that contains two protein subunits (called TFIIE $\alpha$ and TFIIE $\beta$ ). While the archaeal counterpart for TFIIE $\alpha$ (called TFE $\alpha$ ) is known, the counterpart for TFIIE $\beta$ is not.

Blombach et al. have now identified the archaeal counterpart of TFIIE $\beta$ in a species of archaea called Sulfolobus and have renamed it TFE $\beta$. Sulfolobus cells are unable to survive without this protein, which works in a similar way to TFIIE $\beta$ in assisting the RNAP to start transcription. Further analyses show that the TFE $\beta$ protein is actually related to a protein subunit that is unique to RNAP III, another eukarotyic RNAP. Both of these proteins contain clusters of iron and sulphur. Blombach et al. also found that these iron-sulphur clusters enable TFE $\beta$ to bind to its TFE $\alpha$ partner to form a transcription factor that can interact with the RNAP and help it to carry out transcription.

These results suggest that the TFE $\alpha / \beta$ transcription factor found in archaea is likely to resemble the ancestor of the TFIIE transcription factors found in living eukaryotes. This discovery provides new insights in the evolutionary history of both the archaeal and the eukaryotic transcription machineries. DOI: 10.7554/eLife.08378.002

TFIIH (Guzman and Lis, 1999; Kim et al., 2000; Fishburn et al., 2015). However, the dependency on TFIIH changes with DNA template topology. Strand separation and subsequent initiation from linear DNA templates strictly depends on TFIIH, but for some promoters this requirement can be overcome by using negatively supercoiled DNA templates (Parvin and Sharp, 1993). Under these conditions DNA melting is weakly stimulated by TFIIE, an effect that is obscured by the strong melting activity of TFIIH (Holstege et al., 1995). Since TFIIH is not strictly essential for RNAPII initiation per se, similar molecular mechanisms are likely to operate during open complex formation of archaeal and eukaryotic RNAPs. The archaeal model systems provide a distinct advantage in this respect allowing us to study how TFIIE/TFE facilitates DNA melting in the absence of a TFIIH-like factor.

Archaea, like bacteria, utilise one type of RNAP to execute their genetic programmes, while the transcription space of eukaryotes is partitioned into distinct and non-overlapping subsets of genes that are transcribed by 3 and 5 specialised types of RNAPs in animals and plants, respectively. The common past of all types of nuclear eukaryotic RNAP systems is reflected in the sequence, structure and function of RNAP subunits and associated basal transcription factors (Vannini and Cramer, 2012). TFIIE is a prominent example of this relationship. The human RNAPIII subunits hRPC62/39 (C82/34 in yeast) are positioned at the periphery of RNAPIII nearly identical to the binding site of TFIIE on RNAPII (Vannini and Cramer, 2012). TFE $\alpha$, TFIIE $\alpha$ and hRPC62 contain homologous winged helix (WH) domains (Carter and Drouin, 2010; Lefèvre et al., 2011) (Figure 1A). TFIIE $\beta$ and hRPC39 both contain tandem WH domains suggesting their paralogous nature (Vannini and Cramer, 2012), although no significant sequence homology was found (Carter and Drouin, 2010). The origin and evolution of these factors and their role in the multiplication of parallel transcription systems in eukaryotes has remained elusive.

All crenarchaeal and most euryarchaeal genomes encode a gene that bears resemblance to the RNAPIII subunit hRPC39 (Blombach et al., 2009). Two regions of this gene show homology to two separate and distinct domains of eukaryotic hRPC39 proteins, namely the second of its three WH domains and a C-terminal domain that includes four highly-conserved cysteine residues (Figure 1A). 
A

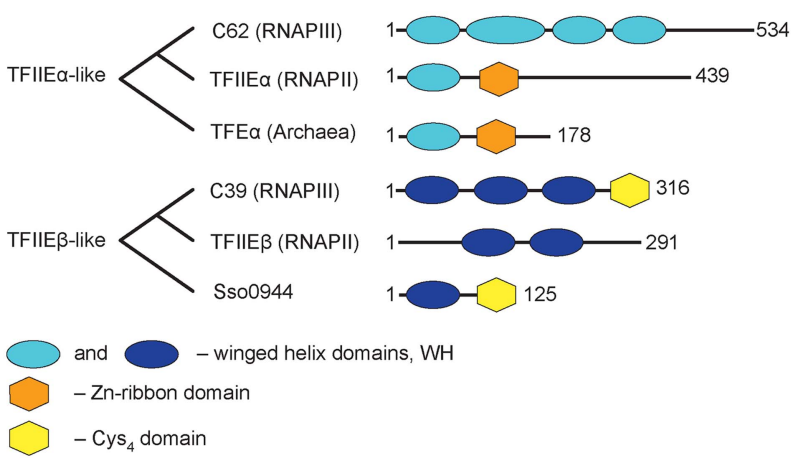

B

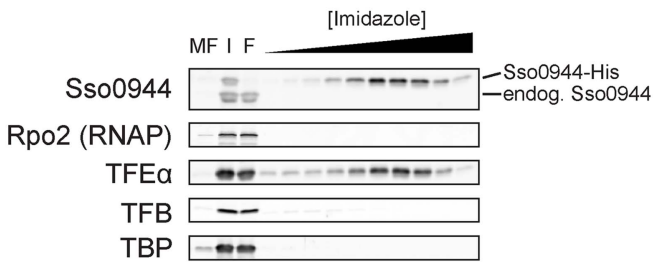

C

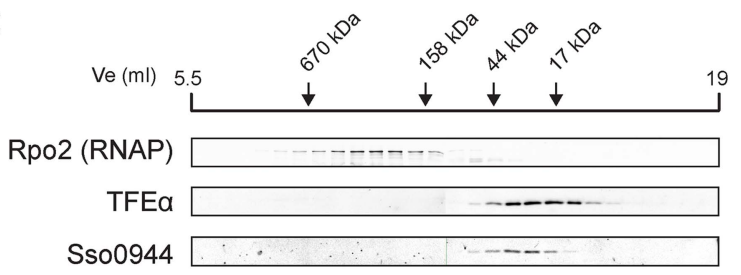

D

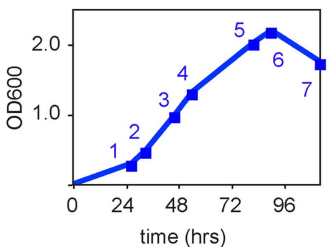

TFB \& Alba

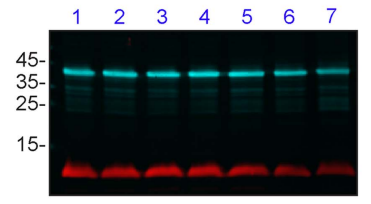

Sso0944 \& Alba

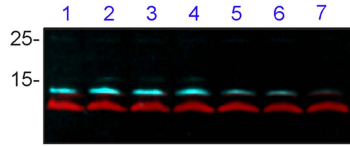

TFEa \& Alba
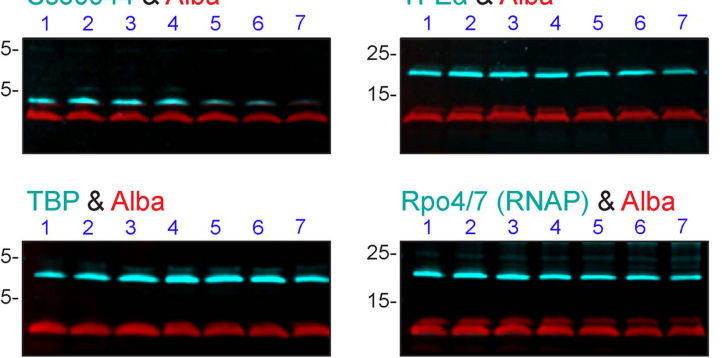

Figure 1. Identification of a dimeric TFE factor in S. solfataricus. (A) Conserved and acquired domains of TFIIE $\alpha$ and TFIIE $\beta$-related proteins in Eukaryotes and Archaea derived from (Blombach et al., 2009; Vannini and Cramer, 2012). (B) Homologous expression and nickel affinity purification of Sso0944 as C-terminal His10-tag fusion. Immunodetection was used to detect co-purification of Sso RNAP (subunit Rpo2) and the basal transcription factors. MF-membrane fraction. I_input (soluble fraction) loaded onto column. F-flow-through fraction. (C) Immunodetection of RNA polymerase (RNAP), TFE $\alpha$ and Sso0944 (TFE $\beta$ ) in S. solfataricus P2 cell lysate fractionated by size exclusion chromatography. (D) Multiplex immunodetection of Sso RNAP (stalk subunits Rpo4/7) and the basal transcription factors in S. solfataricus P2 during different growth phases. Samples were taken at the indicated time points (blue). $18 \mu \mathrm{g}$ of lysed cells (total soluble protein content) was loaded into each lane. Immunodetection of the chromatin protein Alba served as loading control. Immunodetection of Rpo4/7 yielded a strong signal for Rpo7 at around 20 kDa, but only faint signal for Rpo4 (around 13 kDa). The experiment was carried out in triplicate and a typical result is shown.

DOI: 10.7554/eLife.08378.003

The following figure supplements are available for figure 1:

Figure supplement 1. Sso0944 and TFE $\alpha$ form a dimeric complex.

DOI: 10.7554/eLife.08378.004

Figure supplement 2. Quantitative immunodetection of TFE $\alpha, \operatorname{TFE} \beta$ and TBP in S. solfataricus P2 cell lysates during exponential growth phase ( $\mathrm{n}=3$ ). DOI: 10.7554/eLife.08378.005

We have conducted a comprehensive structure-function characterisation of the archaeal hRPC39like gene product using both in vivo and in vitro approaches. Our results show that the hRPC39-like gene from the archaeon Sulfolobus solfataricus is the bona fide homologue of eukaryotic TFIIE $\beta$.

\section{Results}

\section{The archaeal hRPC39-like protein is not an RNAP subunit but forms a complex with TFE $\alpha$}

We chose the hRPC39 homologue Sso0944 from the archaeon S. solfataricus (Sso) as model protein because the gene is a good representative of its kind (Blombach et al., 2009). To identify interaction partners of Sso0944 we expressed His-tagged Sso0944 in S. solfataricus M16 and probed the presence of co-purifying components of the basal transcription apparatus following metal-affinity 
chromatography by immunodetection. While we found no evidence that the RNAP, TBP or TFB1 copurified with Sso0944, TFE $\alpha$ co-eluted with Sso0944, indicating that TFE $\alpha$ and Sso0944 are associated in vivo (Figure 1B). Sypro Ruby-stained SDS-PAGE of the affinity-purified material demonstrates that the polypeptides form a dimeric complex, that is, their association is not dependent on additional factors (Figure 1-figure supplement 1). To rule out the possibility that the affinity tag of Sso0944 prevented its stable association with the RNAP we fractionated a wild type $S$. solfataricus P2 cell lysate by size exclusion chromatography and analysed the fractions using immunodetection. The elution profile of endogenous Sso0944 overlapped with that of TFE $\alpha$ consistent with a heterodimeric TFE $\alpha /$ Sso0944 complex of $36.1 \mathrm{kDa}$. The elution profile of TFE $\alpha$ was somewhat broader indicating that part of TFE $\alpha$ might be present in the monomeric form. RNAP eluted in earlier fractions corresponding to its molecular weight of approximately $400 \mathrm{kDa}$ (Figure 1C). As Sso0944 is not stably incorporated into RNAP in contrast to the related RNAPIII subunit hRPC39, but rather forms a complex with TFE $\alpha$, we renamed the archaeal protein TFE $\beta$.

\section{TFE $\beta$ is essential for cell viability and depleted during stationary phase}

Like most genes that encode basic components of the transcription apparatus, both RPC 34 and TFA2, the genes encoding C34 and Tfa2, respectively, are essential for cell viability in Saccharomyces cerevisiae (Stettler et alo, 1992; Feaver et alo, 1994). In order to test whether the gene encoding TFE $\beta$ is essential in Sulfolobus we attempted to delete the gene (Saci_1342) in the uracil-auxotroph Sulfolobus acidocaldarius strain MW001 using a pop-in/pop-out strategy and selection marker pyrEF (Wagner et alo, 2012). Following genomic integration of the Saci_1342 deletion construct, counterselection using 5 -fluoroorotic acid all clones reverted to wild type via reciprocal excision (80 clones tested) (Table 1 and Table 1-source data 1). In contrast, deletion of Saci_1342 was readily achieved in strain MW001 Saci_1162::Saci_1342 where we introduced a second copy of Saci_1342 replacing the non-essential gene Saci_1162 (4 out of 20 clones) (Table 1 and Table 1-source data 1). This ultimately demonstrates that the gene encoding TFE $\beta$ is essential in $S$. acidocaldarius.

In order to compare and characterise the steady-state levels of TFE $\alpha$ and TFE $\beta$ during exponential and stationary growth of $S$. solfataricus we carried out quantitative Western blotting. TFE $\alpha$ and TFE $\beta$ levels are near stoichiometric during exponential growth $(24 \pm 3 \mathrm{pmol} / \mathrm{mg}$ soluble protein and $27 \pm 3 \mathrm{pmol} / \mathrm{mg}$ soluble protein, respectively) and about sevenfold lower than TBP levels $(184 \pm 17 \mathrm{pmol} / \mathrm{mg})$ (Figure 1-figure supplement 2). TFE $\beta$ levels are decreased when cells enter stationary phase while RNAP, TBP, TFB and TFE $\alpha$ remain largely unaffected (Figure 1D). Our results demonstrate that dimeric $\mathrm{TFE} \alpha / \beta$ is the predominant form of the factor in exponentially growing cells, and that the steady-state levels of the complex vary as a function of the growth cycle.

\section{Archaeal TFE $\beta$ and human hRPC39 contain a cubane iron-sulphur cluster} In order to carry out a structure-function analysis of TFE $\alpha / \beta$ we expressed and purified a recombinant form of the TFE $\alpha / \beta$ complex in in Escherichia coli. Concentrated recombinant TFE $\alpha / \beta$ has a dark brown colour and its absorption spectrum displays a shoulder at $410 \mathrm{~nm}$ that is characteristic for iron-sulphur cluster harbouring proteins (Figure 2A). In order to define the origin of this absorption more precisely we recorded continuous-wave electron paramagnetic resonance (cw-EPR) spectra. The profile of the $\mathrm{cW}$-EPR spectrum with a g-value of 2.01, its sensitivity to the reducing agent sodium dithionite and the

Table 1. Genetic experiments showing that the TFE $\beta$ encoding gene Saci_1342 is essential

\begin{tabular}{llll}
\multirow{2}{*}{ Parental strain } & $\begin{array}{l}\text { Plasmid integration relative } \\
\text { to Saci_1342 }\end{array}$ & $\begin{array}{l}\text { Number of } \\
\text { clones tested }\end{array}$ & $\begin{array}{l}\text { Clones with Saci_1342 } \\
\text { deletion obtained }\end{array}$ \\
\hline MW001 & upstream & 40 & 0 \\
\cline { 2 - 4 } & downstream & 40 & 0 \\
\hline MW001 Saci_1162::Saci_1342 & upstream & 10 & 4 \\
\cline { 2 - 4 } & downstream & 10 & 0 \\
\hline
\end{tabular}

DOI: $10.7554 /$ LLife.08378.006

Source data 1. Genetic experiments showing that the TFE $\beta$ encoding gene Saci_1342 is essential DOI: 10.7554/eLife.08378.007 
A

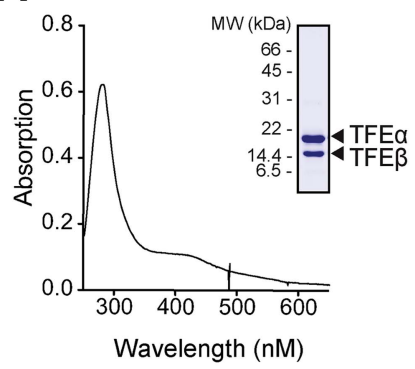

D

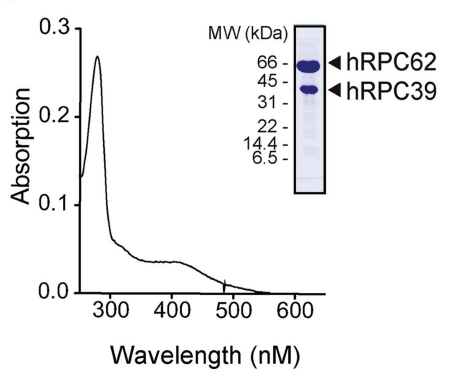

B

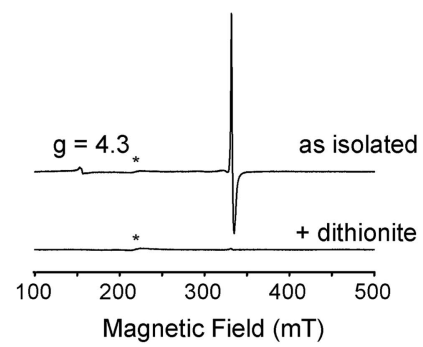

E

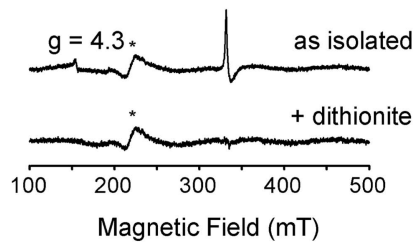

G

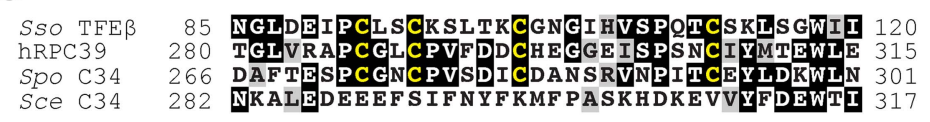

C

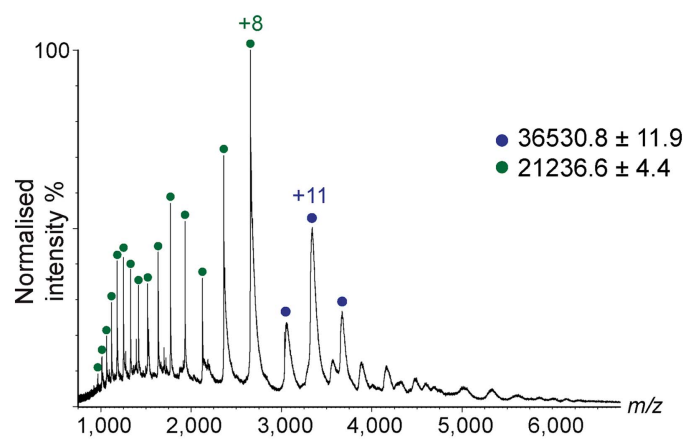

$\mathbf{F}$

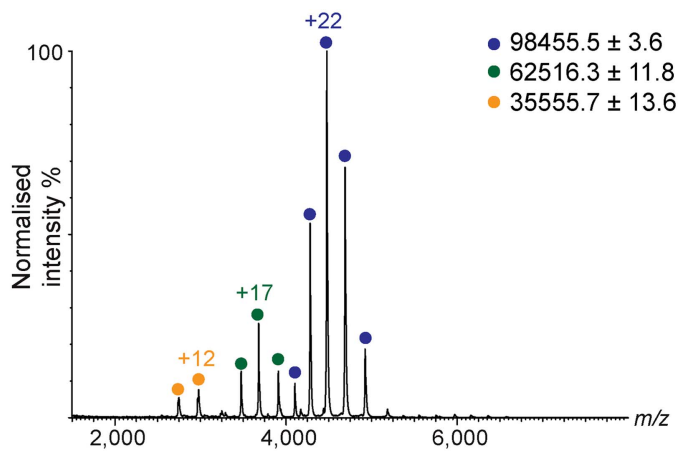

Figure 2. The C-terminal domain of Sso TFE $\beta$ harbours a 4Fe-4S cluster that is conserved in human RNAPIII subcomplex hRPC62/39. (A) UV-vis spectrum of TFE $\alpha / \beta$-His. (B) Cw-EPR spectra of TFE $\alpha / \beta$ prepared in the presence of $1 \mathrm{mM} \mathrm{DTT}$ with or without the addition of $20 \mathrm{mM} \mathrm{Na}$-dithionite. Besides the main $[3 \mathrm{Fe}-4 \mathrm{~S}]^{+}$cluster signal at $\mathrm{g}=2.01$, a small amount of spurious high spin $\mathrm{Fe}^{3+}$ at $\mathrm{g}=4.3$ was also detected. The asterisks denote a background signal. (C) Nano-electrospray ionization ( $n E S I)$ mass spectrum of TFE $\alpha / \beta$-His. Filled circles indicate the different charge state series: TFE $\alpha / \beta$-His $+Z n$ ion $+4 F e-4 S$ cluster (blue), TFE $\alpha+$ Zn ion (green). (D) UV-vis spectrum of recombinant human RNAP III subcomplex hRPC62/C39. (E) CW-EPR spectra of hRPC62/39 prepared in the presence of the reducing agent Na-dithionite. The asterisks denote a background signal. (F) nESI mass spectrum of recombinant human hRPC62/C39. Filled circles indicate the different charge state series: hRPC62/C39 complex + 4Fe-4S cluster (blue), monomeric hRPC62 (green),

monomeric hRPC39 (yellow). (G) Sequence alignment of the C-terminal domains of S. solfataricus TFE $\beta$ (gene id: 1455187), H. sapiens hRPC39 (gene id: 10621), Schizosaccharomyces pombe C34 (gene id: 2538992), and S. cerevisiae C34 (gene id: 855737). Identical and similar residues are shaded in black and grey, respectively. The four cysteine residues coordinating the FeS cluster are highlighted in yellow.

DOI: 10.7554/eLife.08378.008

The following source data and figure supplements are available for figure 2:

Source data 1. Theoretical and experimentally calculated masses of proteins and protein complexes. DOI: 10.7554/eLife.08378.009

Figure supplement 1. Temperature dependence of the cw-EPR spectra of Sso TFE $\alpha \beta$ and hRPC62/39. DOI: 10.7554/eLife.08378.010

Figure supplement 2. Nano-electrospray ionization mass spectrum of human hRPC62/C39 (top).

sharp decrease in signal at temperatures above $20 \mathrm{~K}$ are consistent with a signal corresponding to a cubane $[3 \mathrm{Fe}-4 \mathrm{~S}]^{+}$cluster (Figure 2B and Figure 2-figure supplement 1). Double integration and comparison with a spin standard indicate a low cluster-occupancy of approximately $4 \%$, which is in contrast to the strong $410 \mathrm{~nm}$ signal in the absorption spectrum. It is common that before reduction aerobically prepared proteins containing [4Fe-4S] clusters display a [3Fe-4S] ${ }^{+}$signal due to oxidative impairment (Beinert et al., 1996). Addition of a reducing agent often results in the disappearance of the signal of the $[3 \mathrm{Fe}-4 \mathrm{~S}]^{+}$cluster concomitant with the appearance of the signal from the intact $[4 \mathrm{Fe}-4 \mathrm{~S}]^{+}$cluster. Here, however, only the former effect is observed: [4Fe-4S] ${ }^{2+}$ clusters may not be reduced-and remain EPR-silent-if the redox potential is lower than that of the reducing agent 
(dithionite), or if the rate of reduction is too slow. Alternatively the reduced [4Fe- $4 \mathrm{~S}]^{+}$cluster could be present in a high spin state, which could give rise to a signal too broad to be detected. To investigate the presence of an EPR-silent [4Fe-4S $]^{+}$cluster, we recorded native mass spectra (MS) of TFE $\alpha / \beta$ (Figure 2C and Figure 2-source data 1). The spectrum contained two major species one with a mass of $21,236.6 \mathrm{Da}$ and the other with a mass of $36,530.8 \mathrm{Da}$. The former corresponds to the expected mass for TFE $\alpha$ with a $\mathrm{Zn}^{2+}$ ion bound by its zinc ribbon (ZR) domain while the later corresponds to the mass of the TFE $\alpha / \beta$ harbouring a $\mathrm{Zn}^{2+}$ ion and a $[4 \mathrm{Fe}-4 \mathrm{~S}]^{2+}$ cluster. Given that the TFE $\alpha \mathrm{ZR}$ harbours a $\mathrm{Zn}^{2+}$ ion, the $[4 \mathrm{Fe}-4 \mathrm{~S}]^{2+}$ cluster must be coordinated by the four cysteines in the $\mathrm{C}$-terminal domain of TFE $\beta$. As this domain is highly conserved in human hRPC39 (Figure 2G), we examined the human protein for the presence of an equivalent FeS cluster. Similar to archaeal TFE $\beta$, the human hRPC62/C39 complex shows an absorption spectrum with a shoulder at $410 \mathrm{~nm}$ (Figure 2D); its $\mathrm{cw}$-EPR signature is characteristic for a [3Fe-4S] $]^{+}$cluster with low occupancy $(<3 \%)$ (Figure $2 \mathrm{E}$ and Figure 2-figure supplement 1). The native mass spectrum this time revealed three major species with masses of 35,555.7, 62,516.3 and 98,455.5 Da corresponding to hRPC39, hRPC62 and hRPC62/C39 complex bound to a $[4 \mathrm{Fe}-4 \mathrm{~S}]^{2+}$ respectively (Figure $2 \mathrm{~F}$ ). Tandem MS experiments revealed that the cluster was bound to hRPC39 (Figure 2-figure supplement 2 and Figure 2-source data 1).

In summary, our results reveal the presence of an [4Fe-4S] cluster in the C-terminal domains of archaeal TFE $\beta$ and the human RNAPIII subunit hRPC39, thus demonstrating that this feature has been conserved through evolution. The four cysteines coordinating the [4Fe-4S] cluster show high conservation in TFE $\beta / h R P C 39$ homologs with some exceptions such as haloarchaea (Blombach et al., 2009) and the yeast S. cerevisiae C34, where all four cysteine residues have been lost (Figure 2G).

\section{The FeS cluster is required for TFE $\alpha \beta$ dimerisation}

TFE $\alpha$ and $\beta$ both have a bipartite domain architectures consisting of a WH and a ZR domain, and a WH and FeS domain, respectively (Figures 1A, 3A). In order to characterise the interaction network between the domains in the TFE $\alpha / \beta$ heterodimer, we tested the dimerization properties of domain deletion and substitution variants using a metal affinity co-purification approach and a His-tagged TFE $\beta$ variant (Figure 3B). The input fractions show that all mutant variants were expressed in a soluble and heat-stable form with the exception of TFE $\beta \Delta 1-84$. The TFE $\alpha$ WH domain is essential for dimerization ( $\Delta 1-110)$, while the ZR domain is dispensable ( $\Delta 114-147$, Figure 3B). However, the WH alone is not sufficient for dimerization ( $\Delta 111-178)$, but requires the C-terminal tail $(\Delta 148-178)$ for interaction with TFE $\beta$. The ZR domain of Sso TFE $\alpha$ includes only three of the four conserved cysteine residues that coordinate the $\mathrm{Zn}$ ion in archaeal TFE $\alpha$ and eukaryotic TFIIE $\alpha$. In line with the ZR being dispensable, mutation of the unpaired cysteine in TFE $\alpha$ (C117S) does not impair complex formation with TFE $\beta$ (Figure 3B). In yeast TFIIE, the $\mathrm{N}$-terminal tip of $\alpha$-helix 3 of the Tfa1 WH domain is essential for dimerization (Grünberg et al., 2012). We tested whether the corresponding region in the TFE $\alpha$ WH domain is essential for TFE $\alpha / \beta$ dimerization by introducing point mutations (K46E, D49T, R51E/K52E) or deleting the entire region $(\Delta 46-52)$ (Figure $3 \mathrm{~B}$ and Figure 3-figure supplement 1 ). None of these mutations abrogated dimerization with TFE $\beta$, which reflects that the interaction network differs between yeast TFIIE and TFE $\alpha / \beta$.

We subsequently determined the TFE $\beta$ domains required for TFE $\alpha$ binding. As the exact domain boundary in TFE $\beta$ is unknown we tested different deletion variants including or excluding linker residues 74-84 in dimerization experiments. The WH domain of TFE $\beta$ is not required for dimerization $(\Delta 1-73)$, while the FeS domain is vital ( $\Delta 85-125$, Figure $3 C)$. In order to investigate the role of the FeS cluster-chelating cysteine residues in TFE $\beta$ we produced cysteine to serine mutants at positions C92, C95, C101 and C112. Following metal affinity chromatography of TFE $\alpha / \beta$, the C92S, C95S and C112S variants rapidly lost the FeS cluster based on the absorbance spectra (Figure 4). Concomitant with the loss of the Fe-S cluster no complexes with TFE $\alpha$ were obtained. In contrast, the fourth mutation C101S had little effect on FeS cluster stability, yield and dimerization.

In summary, our results identify the TFE $\beta$ FeS domain as essential part of the TFE $\alpha / \beta$ dimerization interface that comprises also the TFE $\alpha \mathrm{WH}$ and tail domains. These are sufficient to form a minimal $\mathrm{TFE} \alpha / \beta$ complex (Figure 3D).

\section{TFE $\alpha / \beta$ binds to the RNAP clamp}

Methanocaldococcus jannaschii TFE $\alpha$ binds the RNAP in a bidentate fashion, the WH domain interacts with the tip of the RNAP clamp coiled coil while the ZR domain locates to the base of the clamp and the 
A
SsoTFEa
30
60
90

1 MVNAEDLFINLAKSLLGDDVIDVLRILLDKGTEMTDEE IANQLNIKVNDVRKKLNLLEEQGFVSYRKTRDKDSGWFIYYWKPNIDQINEI $\Delta 46-52$

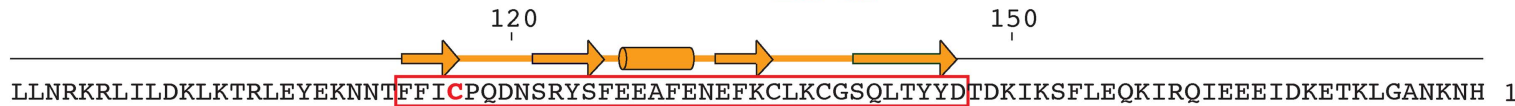

LLNRKRLILDKLKTRLEYEKNNTFFICPQDNSRYSFEEAFENEFKCLKCGSQLTYYDTDKIKSFLEQKIRQIEEEIDKETKLGANKNH 178 $\triangle 114-147$

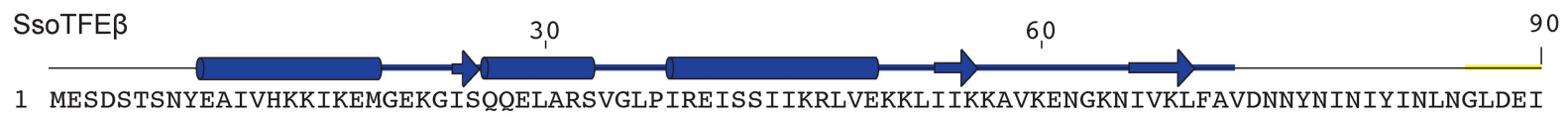

120

PCLSCKSLTKCGNGIHVSPQTCSKLSGWIIEKALS 125

B

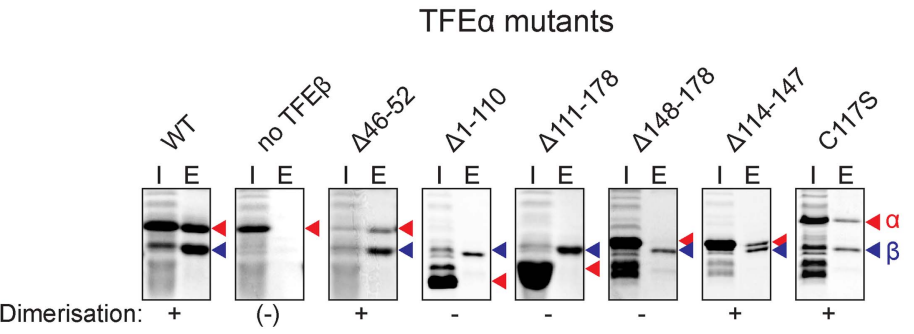

C

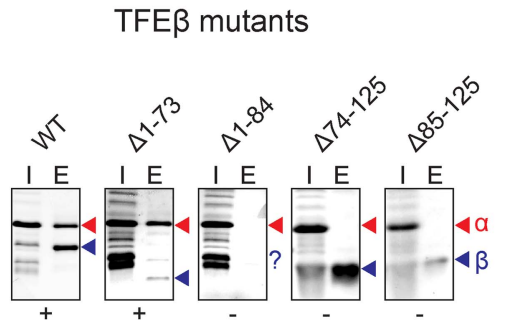

D

Minimal complex

Figure 3. Characterisation of the TFE $\alpha / \beta$ heterodimerization interface. (A) Amino acid sequences and secondary structure of TFE $\alpha$ and TFE $\beta$. The $\alpha$-helices in the TFE $\alpha$ WH domain are numbered according to (Meinhart et al., 2003). Deletion (red boxes) and substitution deletions (highlighted in red) are indicated. (B, C) TFE $\alpha / \beta$ interaction analysis using co-purification of TFE alpha and his-tagged TFE beta. TFE $\alpha$ (B) and TFE $\beta$-His (C) substitution- and domain deletion variants were co-expressed in E. coli and purified using Nickel-affinity chromatography. Input (heat-stable cell lysate, 25\%) (I) and elution fractions (E) were analysed by SDS-PAGE and Coomassie staining. Red and blue triangles indicate the position of the respective mutant variants of TFE $\alpha$ and TFE $\beta$-His, respectively. The question mark denotes that the TFE $\beta \Delta 1-84$ variant is probably instable. Note that the TFE $\alpha$ expression levels are higher than TFE $\beta$, and that the expression levels of TFE $\beta$ mutants are lower than WT, which in some cases make it difficult to discern in the input fractions.

(D) Minimal heterodimeric TFE $\alpha / \beta$ complex consisting of TFE $\alpha \Delta 114-178$ and TFE $\beta \Delta 1-73$.

DOI: 10.7554 /eLife.08378.012

The following figure supplement is available for figure 3 :

Figure supplement 1. Additional mutants of the N-terminal tip of $\alpha$-helix 3 of TFE $\alpha$ and their effect on dimerization with TFE $\beta$ DOI: 10.7554/eLife.08378.013

stalk module (Grohmann et al., 2011). In order to characterise the binding characteristics of TFE $\alpha / \beta$ to Sso RNAP we produced a recombinant RNAP clamp analogously to (Martinez-Rucobo et al., 2011). Gel filtration elution profiles show that TFE $\alpha$ forms a stable complex with the recombinant RNAP clamp since both proteins eluted in earlier fractions corresponding to a larger size (Figure 5). The elution of both proteins was slightly asymmetrical, possibly due to partial dissociation during chromatography. Similarly dimeric TFE $\alpha / \beta$ forms a stable complex with the clamp since all three proteins co-eluted in a symmetrical fashion. Deletion of the TFE $\alpha$ ZR domain (TFE $\alpha \Delta Z R$, residues 114-147) leads to loss of complex formation. In contrast, deleting the TFE $\beta$ WH domain (TFE $\beta \Delta W H$, residues 1-73) does not impair binding (Figure 5) which suggests that the TFE $\beta$ WH domain does not contribute to RNAP binding.

In summary, both the TFE $\alpha$ WH and ZR domains are required to anchor TFE to the RNAP clamp. The TFE $\beta$ FeS-domains further stabilizes the complex, whereas the TFE $\beta$ WH domain appears not to be involved.

\section{TFE $\alpha / \beta$ stabilizes the PIC and facilitates DNA melting}

We developed an electrophoretic mobility shift assay (EMSA) to monitor the formation of the preinitiation complex (PIC) on the viral SSV1 T6 promoter (Qureshi et al., 1997; Werner and 

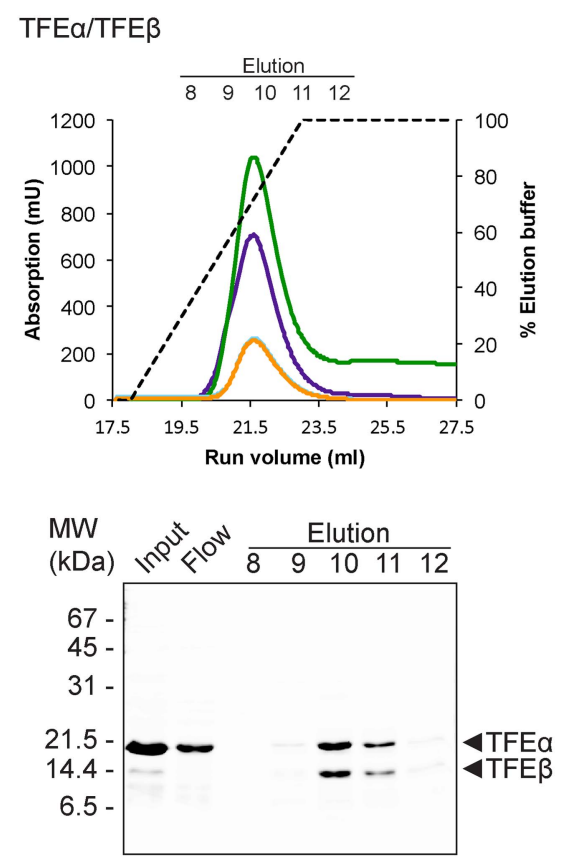

TFEa/TFE $\beta$ C101S
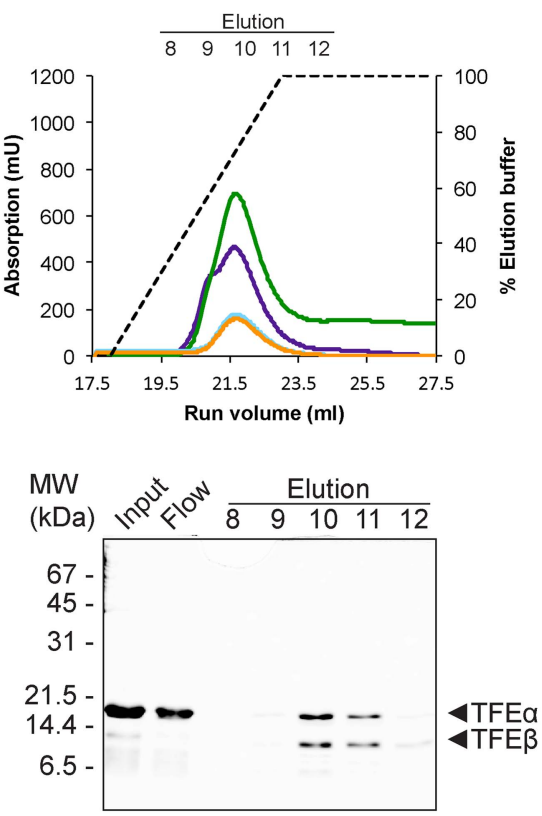

\section{TFEa/TFE $\beta$ C92S}
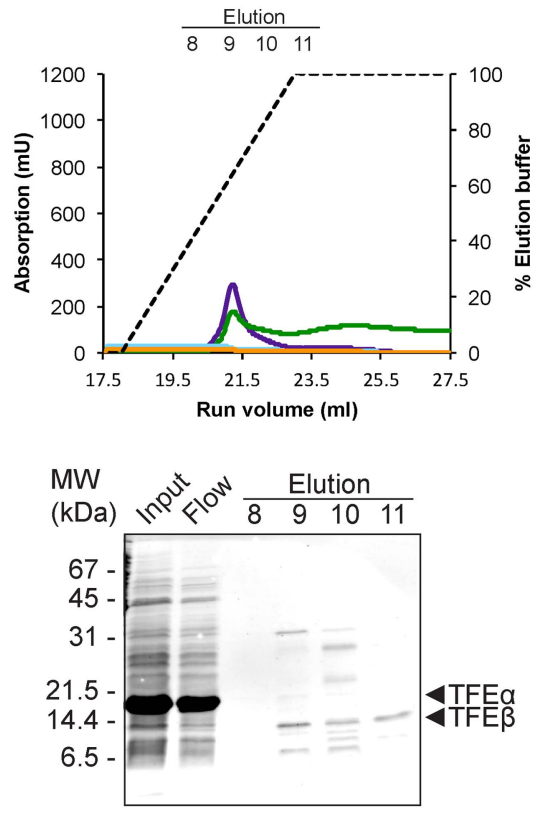

TFE $\alpha / T F E \beta$ C95S
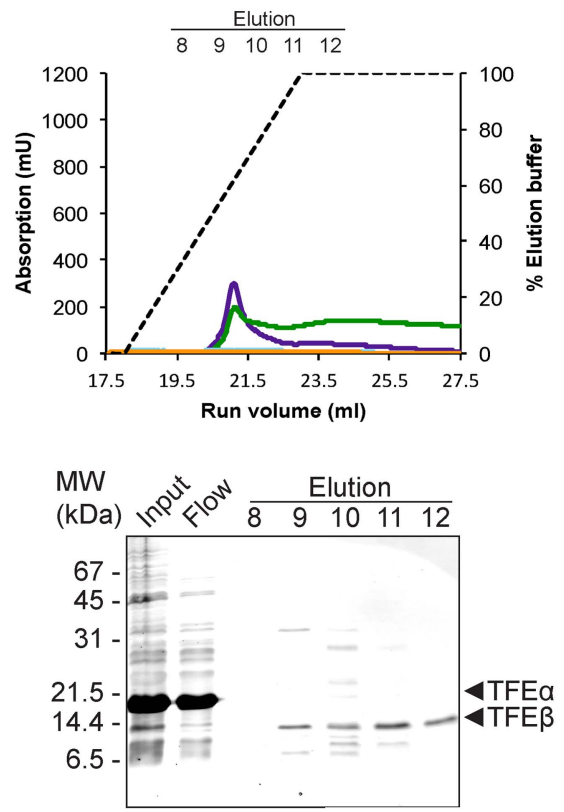

\section{TFE $\alpha /$ TFE $\beta$ C112S}
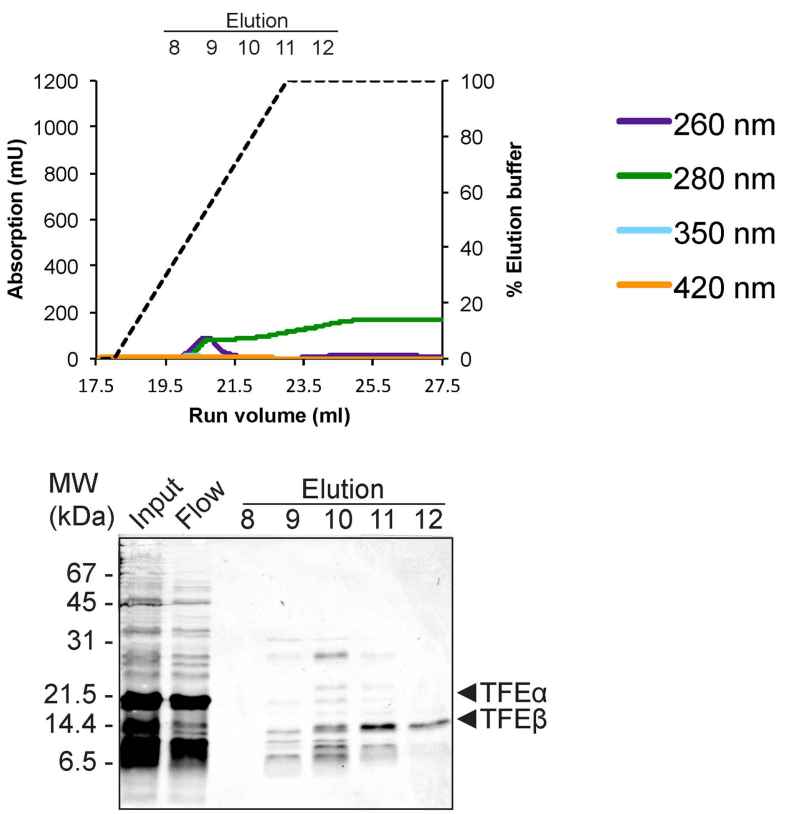

Figure 4. TFE $\alpha \beta$ dimerization depends on the integrity of the Fe-S cluster. Ni-affinity chromatography of TFE $\beta$-His (WT), TFE $\beta$-His C92S, TFE $\beta$-His C95S, TFE $\beta$-His C101S or TFE $\beta$-His C112S co-expressed with TFE $\alpha$. The graphs show the absorption profile monitored at $260 \mathrm{~nm}, 280 \mathrm{~nm}, 350 \mathrm{~nm}$, and $420 \mathrm{~nm}$. Absorption in the visible light range $(350 \mathrm{~nm}$ and $420 \mathrm{~nm}$ ) is indicative for the presence of the Fe-S cluster. The elution fractions analysed by SDS-PAGE are indicated. The panels below show Coomassie-stained SDS-gels with the (heat-stable) input, flow, and elution fractions.

Weinzierl, 2005). Due to the intrinsic instability of the closed Sso PIC we used promoter templates that were pre-melted in the region of -4 to -1 relative to the transcription start site (TSS) (Figure 6A). PIC formation is strictly dependent on both TBP and TFB (Figure $6 B$ ). The addition of TFE $\alpha / \beta$ to the DNA-TBP-TFB-RNAP complex increases the PIC signal in a concentration-dependent fashion with the PIC now appearing in two different species indicating two different conformations. In contrast, 


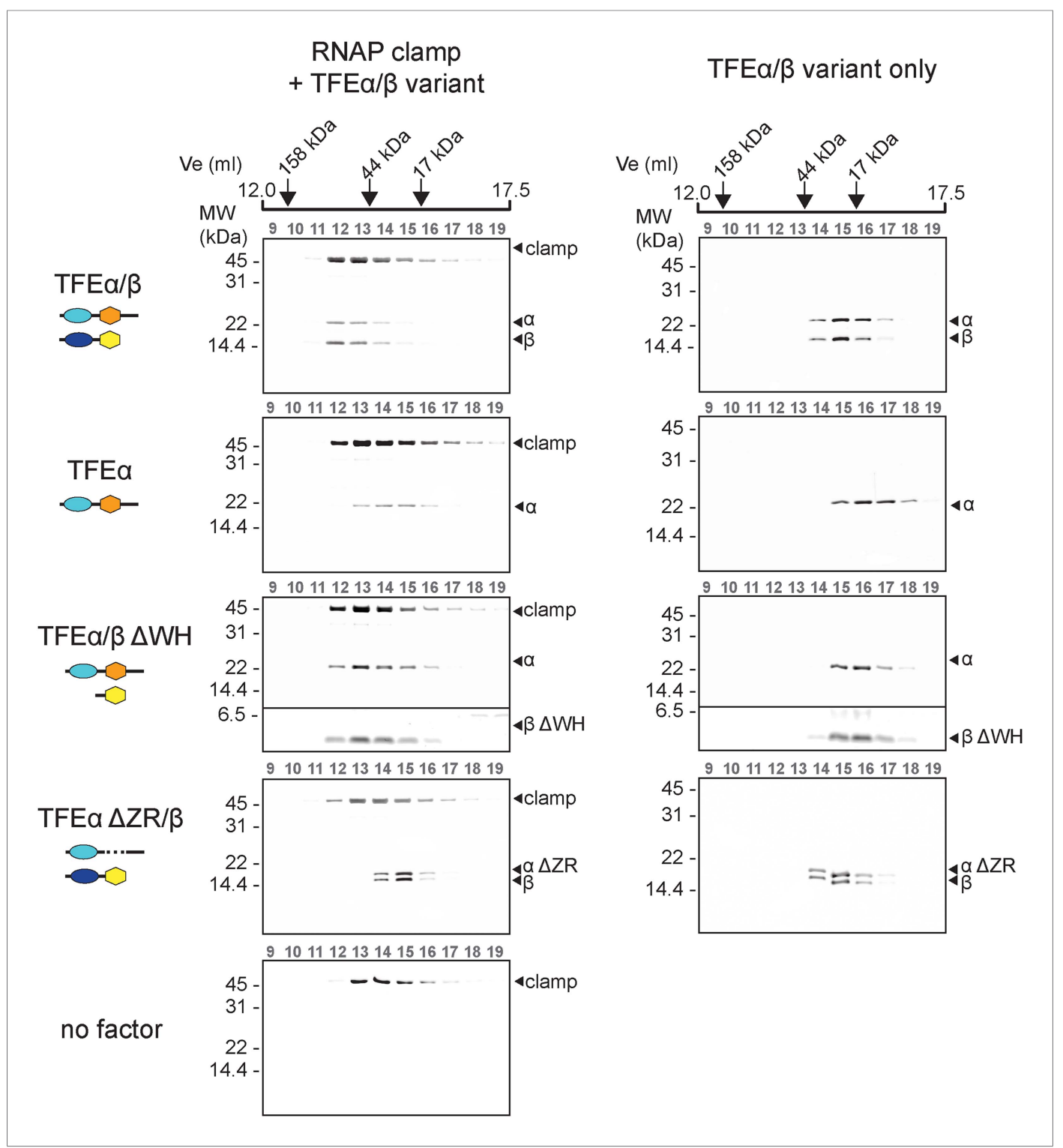

Figure 5. TFE $\alpha / \beta$ forms a stable interaction with the RNAP clamp module. $10 \mu \mathrm{M}$ TFE $\alpha / \beta$-His were incubated together with $10 \mu \mathrm{M}$ recombinant RNAP clamp and the sample was resolved gel filtration. The presence of RNAP clamp and TFE $\alpha / \beta$ in the fractions was determined by SDS-PAGE and silver staining. The position of peaks for gel filtration marker proteins is indicated on top. For experiments with TFE $\alpha / \beta \Delta \mathrm{WH}$ the contrast was enhanced for the lower part of the gel in order to visualize TFE $\beta \Delta \mathrm{WH}$.

DOI: 10.7554/eLife.08378.015

monomeric TFE $\alpha$ had no stimulatory effect on PIC formation (Figure 6B). TFE $\alpha / \beta$ was not able to bind to the dsDNA template directly (Figure 6B and data not shown).

Deletion of the TFE $\alpha$ ZR or the TFE $\beta$ WH domains reduced the stimulation. The former mutation destabilizes the binding to the RNAP, while the latter variant is not impaired in RNAP binding (Figure 5), which suggests that the TFE $\beta$ WH domain plays a role for the stabilisation of the PIC. EMSA supershift experiments validated the incorporation of TFE $\beta$ into the PIC (Figure 6-figure supplement 1).

In order to test the ability of TFE $\alpha / \beta$ to facilitate DNA melting we carried out permanganate footprinting assays to identify T-residues in single-stranded regions within the NTS. The same $-4 /-1$ pre-melted T6 promoter template was used in EMSA and permanganate assays, which accordingly result in a strong signal at -1 . The ternary complex (DNA-TBP-TFB) gives in addition, and to a much lesser extent, a signal at -5 likely due to thermal breathing (Figure $6 \mathrm{C}$ ). The inclusion of RNAP displayed a similar pattern, while the addition of TFE $\alpha / \beta$ led to new strong signals at $-5,-7$ and -12 , 
A

initially meited region

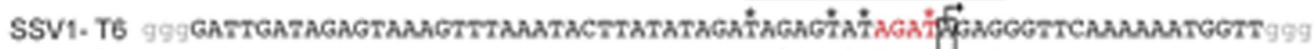

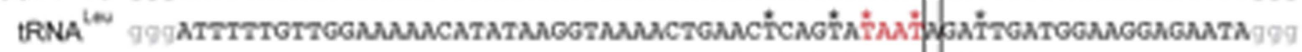

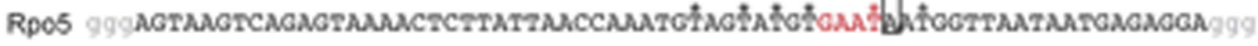

B

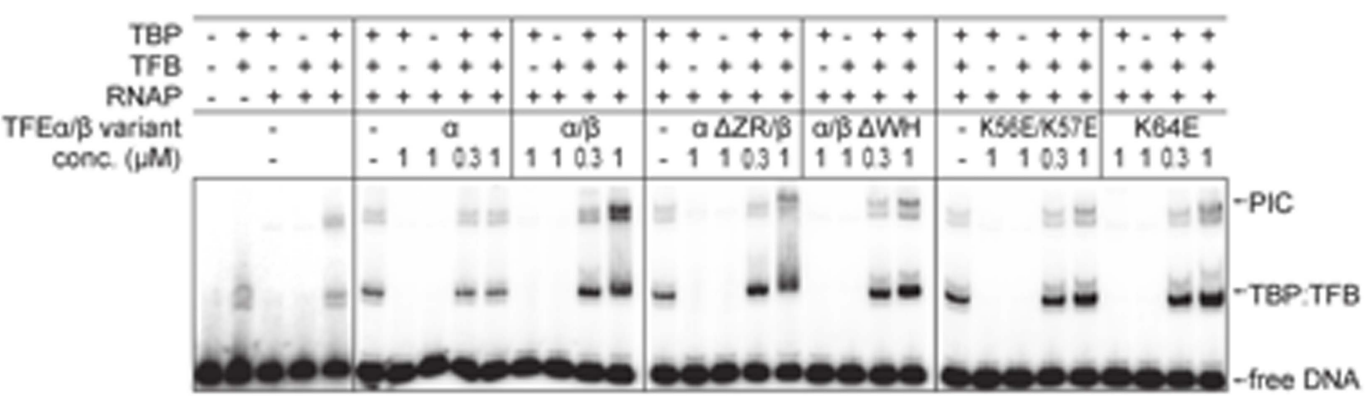

C

SSV1-T6

tRNA ${ }^{\text {in }}$

Rpo5

TFE variant
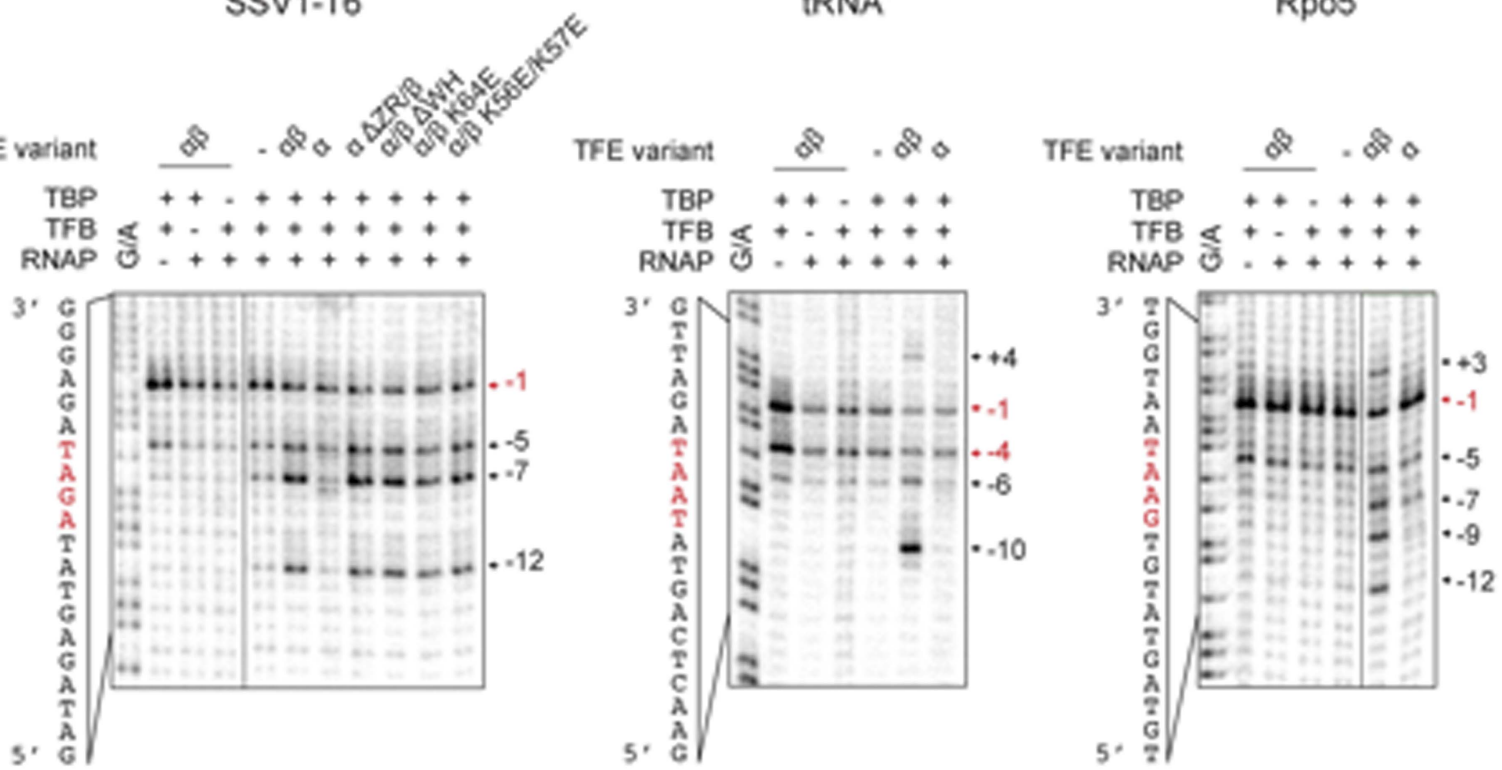

D

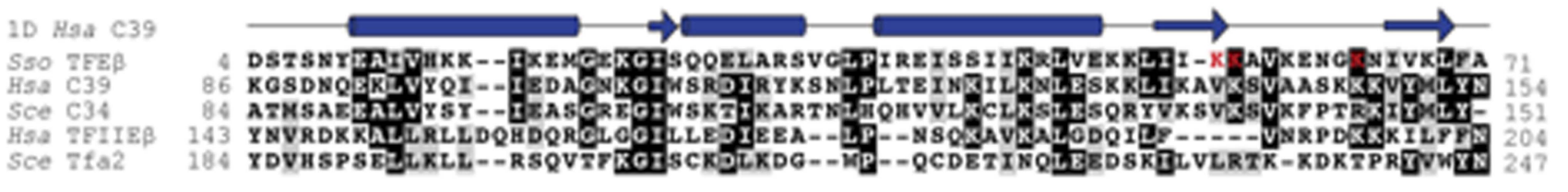

Figure 6. TFE $\alpha / \beta$ increases the stability of the preinitiation complex (PIC) and promotes DNA melting. (A) NTS sequence of the DNA templates for the promoters tested in electrophoretic mobility shift assay (EMSA) and potassium permanganate footprinting assays. The templates incude a $4 \mathrm{nt}$ heteroduplex region (positions -4 to -1 relative to the TSS, in red) that was generated by introducing transition mutations into the template strand Asterisks mark permanganatereactive T residues (see panel C). Additional G residues (in grey) were added to stabilize the termini. (B) PIC formation on the SSV1-T6 promoter in response to TFE $\alpha / \beta$, TFE $\alpha$ and mutant variants using EMSAs. K56E/K57E and K64E denote mutations in the TFE $\beta$ WH. (C) The non-template strand of the SSV1-T6 and Sso Rpo5 and Sso tRNA ${ }^{\text {Leu }}$ promoters was probed in potassium permanganate footprinting assays. The position of reactive T residues is indicated on the right. G/A ladder and the DNA sequence are shown on the left. (D) Sequence alignment of the WH domains of TFE $\beta$ (gene id: 1455187), WH2 of human C39 (gene id: 10621), WH2 of yeast C34 (gene id: 855737), WH2 of human TFIIE $\beta$ (gene id: 2961), and WH2 of yeast Tfa2 (gene id: 853936). Identical and similar residues are shaded in black and grey, respectively. On top of the alignment the secondary structure of WH2 of human C39 (PDB id: 2DK5) is depicted ( $\alpha$-helices as barrels and $\beta$-strands as arrows). Residues K56, K57 and K64 in the WH domain of TFE $\beta$ where mutations were introduced are highlighted in red.

DOI: 10.7554/eLife.08378.016

Figure 6. continued on next page 
Figure 6. Continued

The following figure supplement is available for figure 6:

Figure supplement 1. TFE $\alpha / \beta$ is part of the PIC.

DOI: 10.7554/eLife.08378.017

which reflects that the factor stimulates open complex formation (Figure $6 C$ ). Deletion of the TFE $\beta$ WH or the TFE $\alpha$ ZR domain did not perturb the promoter opening activity of the TFE $\alpha / \beta$ complex, while TFE $\alpha$ was inactive, congruent with its lack of PIC stabilisation (Figure 6C). Similar experiments

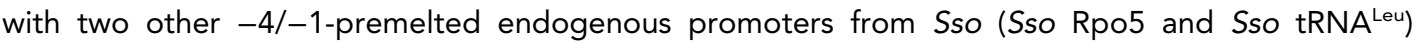
confirmed that TFE $\alpha / \beta$ strongly enhances open complex formation up to position +4 (Figure $6 C$ ). The WH domains of TFE $\beta$, hRPC39/C34 and TFIIE $\beta / T$ fa2 show little conservation, but they commonly carry sets of lysine residues in the wing formed by the two C-terminal $\beta$-strands and the loop between them (Figure 6D) that may play a role in electrostatic interactions with the phosphate-backbone of the NTS. We generated two charge reversal mutations in the TFE $\beta$ WH domain, K56E/K57E and K64E. In EMSA experiments, both mutants showed reduced stimulation in PIC formation when compared to WT $\mathrm{TFE} \alpha / \beta$ (Figure 6B). In permanganate footprinting assays both mutants supported open complex formation similar to WT TFE $\alpha / \beta$ or TFE $\alpha / \beta \Delta \mathrm{WH}$ (Figure $6 \mathrm{C}$ ).

\section{TFE $\alpha / \beta$ stimulates abortive and productive transcription}

In order to test the influence of TFE $\alpha / \beta$ on the formation of the first phosphodiester bond we developed a promoter-dependent dinucleotide extension assay. RNAP is able to add one ATP molecule to an ApG dinucleotide to synthesize an ApGpA trinucleotide in a TBP/TFB factordependent fashion (Figure 7A). This reaction has a low TFB-independent background, but is strictly dependent on the DNA sequence of the promoter since it relies on ApG and ATP (Figure 7A). TFE $\alpha / \beta$ $(1 \mu \mathrm{M})$ stimulates abortive transcription activity on closed promoter templates $(2.3 \pm 0.5$ fold $)$ as well as pre-opened $(1.6 \pm 0.1$ fold) templates (Figure 7A) while no stimulation was observed with TFE $\alpha$ (data not shown).

In order to ascertain the function of TFE $\alpha / \beta$ on productive transcription we fused different promoters to a C-less cassette and carried out transcription assays in the presence of GTP, ATP and UTP. We compared the viral T6 and five cellular promoters of protein encoding genes (Sso EF-1 $\alpha$, Sso SSB, and Sso Rpo5), as well as noncoding RNA genes (Sso tRNA ${ }^{\text {Leu }}$ and 16S/23S rRNA) (Figure 7B). On all tested promoters the addition of TFE $\alpha / \beta$ stimulated transcription by approximately twofold to fourfold, with the strong T6 and 16S/23S rRNA promoters showing a weaker response (Figure 7C and Figure 7-figure supplement 1). These results suggest that the stimulation of transcription by TFE $\alpha / \beta$ is dependent on the sequence of the promoter. Considering that TFE $\alpha / \beta$ stimulates DNA melting we hypothesised that the initially melted region of the promoter $(-12$ to +4$)$ determines the amplitude of the stimulation. We generated hybrid promoters encompassing the TATA-box and surrounding region (position -46 to -13 ) of the weakly stimulated 16S/23S rRNA promoter with the initially melted and transcribed regions of the stronger stimulated Sso tRNA ${ }^{\text {Leu }}(-12$ to +5$)$ or EF- $1 \alpha$ promoters $(-12$ to +7$)$. In absence of TFE $\alpha / \beta$ the hybrid promoters show reduced activity compared to the wild-type rRNA promoter underlining that the initially melted region contributes to the strength of the ribosomal promoter (Figure 7D). In line with our hypothesis TFE $\alpha / \beta$ stimulated transcription on the two hybrid promoters to greater extent when compared to the wild-type rRNA promoter, confirming that the sequence of the initially melted region determines the extent of TFE $\alpha / \beta$ stimulation. Interestingly, both TFE $\beta$ WH deletion and charge reversal mutations, and the TFE $\alpha$ ZR deletion mutants were able to stimulate transcription from the T6 and Rpo5 promoters (Figure 7E and Figure 7-figure supplement 2).

In summary, TFE $\alpha / \beta$ appears as a basal transcription factor that stimulates transcription on mRNA as well as noncoding RNA genes.

\section{Discussion}

\section{TFE $\beta$ and hRPC39 contain structural FeS domains}

We have discovered the TFIIE $\beta$ homologue in archaea, TFE $\beta$, and characterised its structure and function. The gene encoding TFE $\beta$ is essential in $S$. acidocaldarius and most likely this is true for 
A

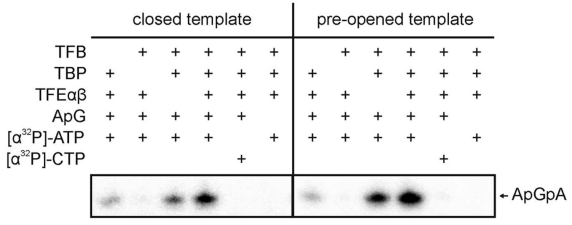

B

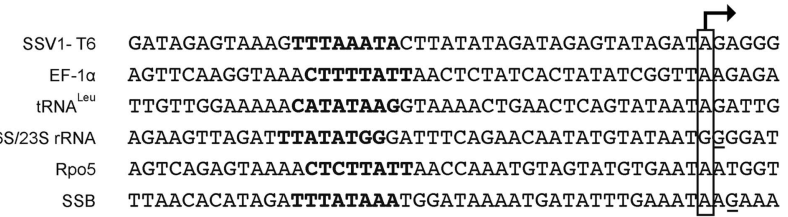

D
C
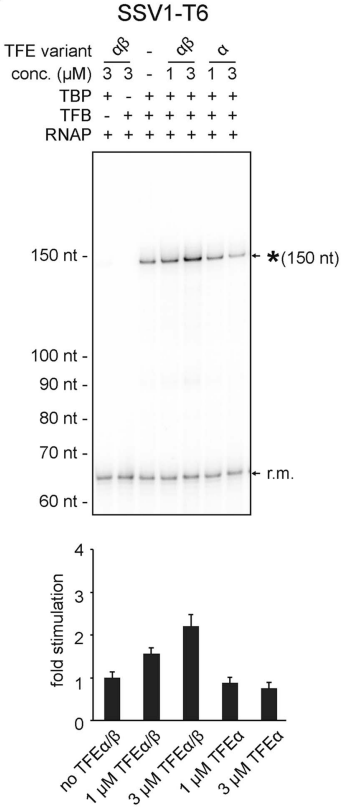

EF-1a
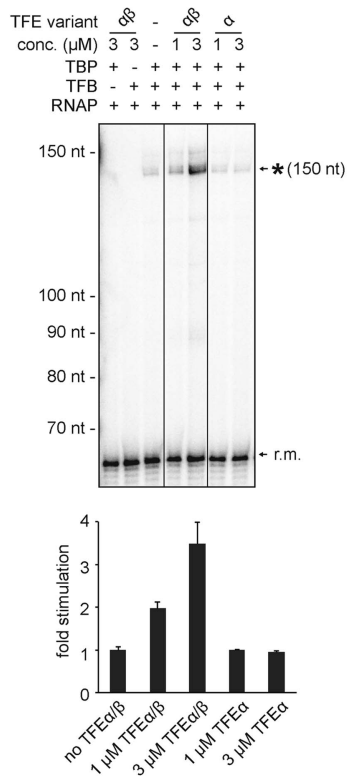

16S/23S rRNA
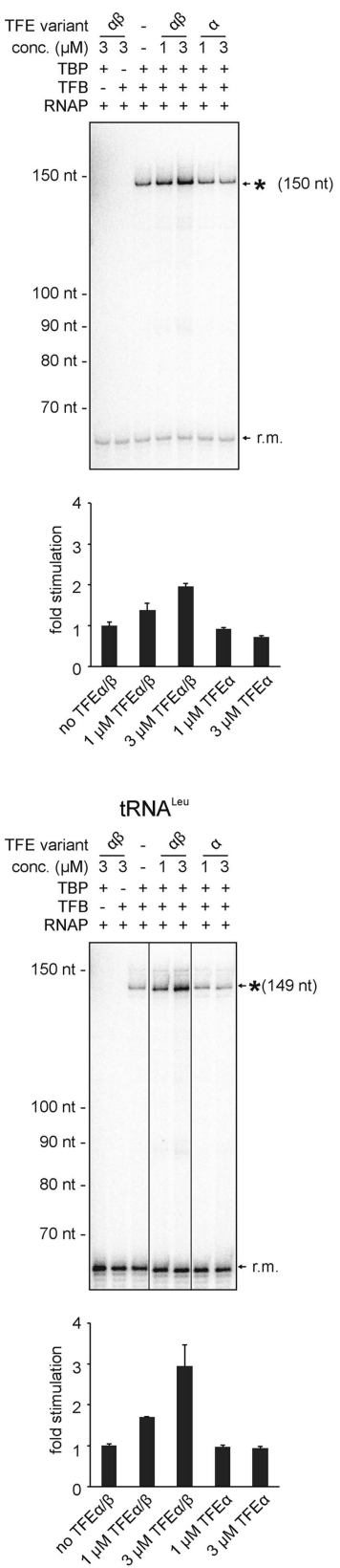
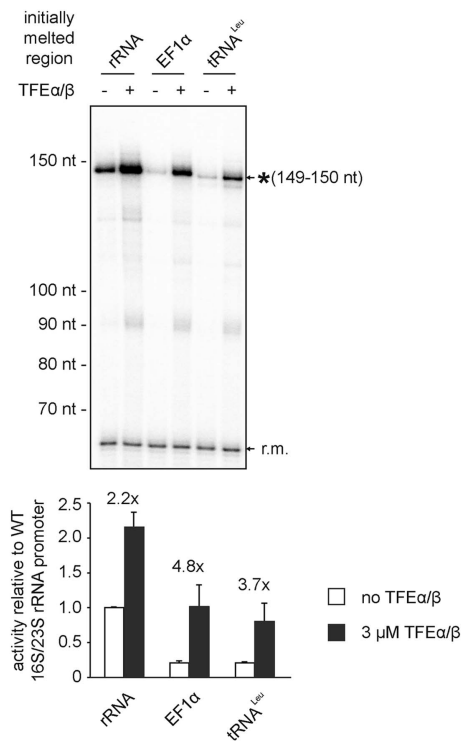

E

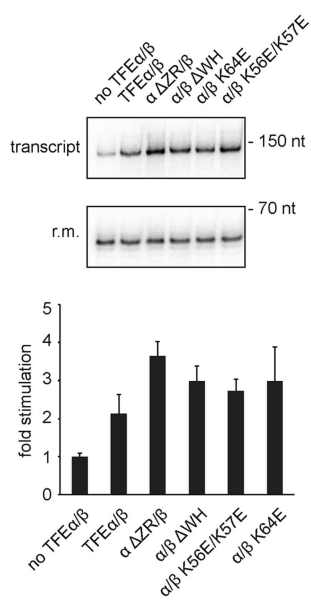

Figure 7. TFE $\alpha / \beta$ stimulates abortive and productive transcription. (A) Abortive transcription assays measuring ApGpA trinucleotide synthesis on $-4 /-1$ heteroduplex and homoduplex SSV1-T6 promoter templates in the presence or absence of $1 \mu \mathrm{M} \mathrm{TFE} \alpha / \beta$. (B) NTS sequence of the different promoters tested in productive transcription assays. The TSS is boxed and putative TATA-boxes are shown in bold. Residues underlined are $\mathrm{C}$ to $\mathrm{G}$ mutations in order to construct a C-less cassette. (C) Productive transcription assays on four different promoters. Circular relaxed plasmids with different $\mathrm{S}$. solfataricus Figure 7. continued on next page 
Figure 7. Continued

promoters fused to C-less cassettes were used as templates. The position of the run-off transcripts (asterisk) and its expected size are indicated. A recovery marker (r.m.) was included. The lower panels show the quantifications of synthesized transcript. (D) Effect of the initially melted region on TFE $\alpha / \beta$ stimulation. Productive transcription assays with hybrid promoters encompassing the TATA-box and surrounding region (position -46 to -13 ) of the weakly stimulated $16 \mathrm{~S} / 23 \mathrm{~S}$ rRNA promoter with the initially melted and transcribed regions of the stronger stimulated tRNA ${ }^{\text {Leu }}(-12$ to +5$)$ or EF-1 $\alpha$ promoters $(-12$ to +7$)$. (E) Effect of TFE $\alpha / \beta$ mutant variants $(3 \mu \mathrm{M})$ on productive transcription on the T6 promoter. The mean of three technical replicates is shown. Error bars depict $1 \times$ standard deviation.

DOI: 10.7554/eLife.08378.018

The following figure supplements are available for figure 7:

Figure supplement 1. Productive transcription assays using the SSB and Rpo5 promoters. DOI: 10.7554/eLife.08378.019

Figure supplement 2. Effect of TFE $\alpha / \beta$ mutant variants on productive transcription on the Rpo 5 promoter. DOI: 10.7554/eLife.08378.020

crenarchaea in general given its strict conservation in the crenarchaeal phylum. On the sequence level TFE $\beta$ is homologous to the eukaryotic RNAPIII subunit hRPC39 and we show that both Sso TFE $\beta$ and human hRPC39 harbour a cubane FeS cluster at their C-termini. Several proteins involved in nucleic acid metabolism have been reported to harbour FeS clusters including multi-subunit RNAPs and TFIIH subunit XPD/Rad3 (White and Dillingham, 2012). Notably, the Sso RNAP subunit Rpo3 includes a structural [4Fe-4S] cluster that is highly stable under aerobic conditions (Hirata et al., 2008). Although TFE $\beta$ could be purified under aerobic conditions with high FeS cluster occupancy, the FeS cluster was lost within a few hours, as apparent from the decolouration of the protein preparation. Furthermore, oxidation with ferricyanide oxidation causes destruction of the TFE $\beta$ cluster (data not shown). This difference between the two FeS clusters in Sso RNAP and TFE $\beta$ likely reflects different functions. The FeS cluster in the catalytic subunit of yeast DNA Polymerase Pol $\delta$ is essential for the interaction with its two auxiliary subunits Pol31 and Pol32 (Netz et al., 2012). Similarly, the FeS cluster is required for TFE $\alpha / \beta$ dimerization and cluster damage results in its dissociation (Figure 4). Our results demonstrate that steady-state levels of TFE $\beta$ are depleted in the stationary phase-the FeS cluster in TFE $\alpha / \beta$ may provide a handle for fast inactivation of TFE $\alpha / \beta$ during stress response. In line with this hypothesis we found oxidative stress induced by hydrogen peroxide leads to rapid depletion of Sso TFE $\beta$ (data not shown).

\section{Structural organisation of TFE $\alpha / \beta$}

The minimal domain requirements for the heterodimerisation of TFE $\alpha$ and $\beta$ encompass the TFE $\alpha$ WH and tail domains, and the TFE $\beta$ FeS domain (Figures 3D, 8A). The position of euryarchaeal TFE $\alpha$ on the RNAP clamp and Rpo4/7 stalk has previously been mapped in the context of the complete PIC from the euryarchaeote M. jannaschii (Grohmann et al., 2011; Nagy et al., 2015). Likewise, Sso TFE $\alpha / \beta$ forms a stable complex with a recombinant RNAP clamp. Our results show that the TFE $\alpha$ ZR makes extensive contacts with the RNAP clamp. The TFE $\beta$ FeS domain stabilizes the binding either by directly interacting with the RNAP clamp or indirectly by altering the conformation of TFE $\alpha$ (Figure 5). In contrast, the TFE $\beta$ WH domain is not involved. The overall structural organisation of the crenarchaeal RNAP-TFE $\alpha / \beta$ ensemble is consistent with PIC models in eukaryotes (RNAPII, He et al., 2013) as well as euryarchaea (Grohmann et al., 2011), and the architecture of RNAPIII (Vannini et al., 2010; Wu et al., 2012). The TFE $\alpha$ WH domain is located on the apex of the RNAP clamp, while its ZR domain projects towards and interacts with the base of the RNAP clamp and stalk module (Grohmann et al., 2011; He et al., 2013) (Figure 8A). The TFE $\beta$ WH domain is likely to project across the DNA binding channel prone to make contacts with the promoter template. The TFE $\beta$ FeS domain may provide additional binding surface to the RNAP clamp.

\section{Molecular mechanisms of TFE}

Following RNAP recruitment to the promoter TFE $\alpha / \beta$ stabilises the PIC (Figure 6B), and this is dependent on the TFE $\alpha$ ZR and the TFE $\beta$ WH domain while TFE $\alpha$ alone has no apparent effect on PIC formation (Figure 6B) similar to its human homologue TFIIE $\alpha$ (Peterson et al., 1991). The proximity of M. jannaschii TFE $\alpha$ WH with the NTS at position -12 (Grohmann et al., 2011) and the location of the 
A

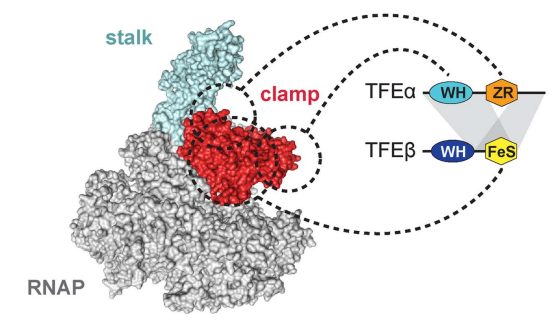

B

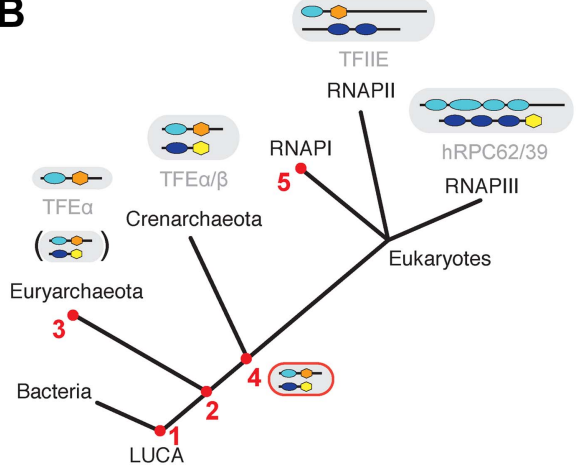

Figure 8. Structure and evolution of TFE $\alpha / \beta$. (A) Model of the dimerization interface of TFE $\alpha / \beta$ and its interaction with the RNAP clamp based on data presented here and elsewhere (Grohmann et al., 2011; Grünberg et al., 2012; He et al., 2013). Grey areas indicate domains of TFE $\alpha$ and $\beta$ that are required for dimerization. Dashed lines indicate interactions between the WH and zinc ribbon domains of TFE $\alpha$ and the RNAP clamp. The FeS domain of TFE $\beta$ stabilizes the interaction with the RNAP clamp and might directly bind. (B) A scenario for the evolution of TFIIE-like proteins in archaea and eukaryotes under assumption of an archaeal origin of eukaryotes according to the 'eocyte' hypothesis (Guy and Ettema, 2011; Williams et al., 2013). Our model includes only Euryarchaeota and Crenarchaeota, the two most studied archaeal clades. Five key steps are indicated with red numbers: 1-TFE $\alpha$ and TFE $\beta$ WH domains are related to bacterial MarR-type WH domains indicating their early evolutionary origin (Aravind et al., 2005; Blombach et al., 2009). 2-The wide distribution of TFE $\alpha$ and TFE $\beta$ encoding genes indicate that both genes date back to the last common archaeal ancestor. 3-In several euryarchaeal species the TFE $\beta$ encoding gene was lost (Blombach et al., 2009) and all biochemically characterised euryarchaeal TFE $\alpha$ appear to function as monomeric factors. Nevertheless, dimeric TFE $\alpha / \beta$ may exist in other euryarchaeal species. 4 -Latest possible emergence of the dimeric TFE $\alpha / \beta$-like factor predating the split of Crenarchaeota and the 'archaeal parent' of eukaryotes. After gene duplication, this precursor evolved to give rise to TFIIE in the RNAPII transcription machinery and the RNAPIII subcomplex hRPC62/39. 5-Eukaryotic RNAPI has lost its dependence on TFIIE-like factors. DOI: 10.7554/eLife.08378.021

human TFIIE $\beta$ WH across the DNA binding channel (He et al., 2013) suggest that TFE $\alpha / \beta$ modulates the handling of the DNA strands by RNAP, for example, during DNA melting and open complex formation. Our permanganate footprinting experiments suggest that TFE $\alpha / \beta$, and not TFE $\alpha$, triggers DNA melting creating a bubble ranging in its extremes from position +4 to -12 position relative to the TSS (Figure 6C). TFE $\alpha / \beta$, but not TFE $\alpha$, stimulates the formation of the first phosphodiester bond in an abortive transcription assay as well as productive transcription. The stimulatory effect of TFE $\alpha / \beta$ on DNA melting and productive transcription appears not to depend on the TFE $\alpha$ ZR and TFE $\beta$ WH domains (Figures 6C, 7E and Figure 7-figure supplement 2). In contrast, in experiments monitoring PIC formation using EMSA that relies to a greater extent on complex stability (as the complexes have to remain intact during electrophoresis), deletion of either TFE $\alpha$ ZR or TFE $\beta$ WH strongly reduces PIC stability (Figure 6B). Similarly, stable interaction with the RNAP clamp depends on the TFE $\alpha$ ZR (Figure 5). This suggests that the core TFE $\alpha / \beta$ composed of the TFE $\alpha \mathrm{WH}$ and the TFE $\beta$ FeS domains can trigger DNA melting and thereby stimulate transcription initiation. The TFE $\alpha$ ZR or TFE $\beta$ WH domains bring addition stability to the complex that becomes crucial in the cellular context. In line with a stabilizing role for the ZR domain, mutation of the cysteine residues in the yeast Tfa1 ZR in vivo confer a thermosensitive phenotype (Kuldell and Buratowski, 1997).

We assessed the impact of TFE $\alpha / \beta$ on the transcription directed by a range of protein-encoding and noncoding RNA promoters. While hRPC39 as part of RNAPIII plays a role in the specific recruitment of RNAPIII to its noncoding RNA gene promoters, the hRPC39-like archaeal TFE $\beta$ clearly acts as a general transcription initiation factor stimulating transcription from all promoters tested. Our results show that the steady state levels of TFE $\beta$ are drastically reduced in stationary phase compared to exponential growth phase, in contrast to other components of the basal Sso transcription apparatus. TFE $\alpha / \beta$ stimulates transcription of different genes to varying extent, dependent on the sequence of the initiatlly melted region within the promoter. Thus, promoters that are strongly 
stimulated by TFE $\alpha / \beta$ could be downregulated in stationary phase, while expression of TFE $\alpha / \beta$ unresponsive (or only mildly stimulated) promoters would be less affected. We do not provide direct evidence for a regulatory role of TFE $\alpha / \beta$, however, our results suggest that TFE $\beta$ has the potential to reprogram transcription in response to different growth phases. TFE $\alpha / \beta$ 's function as basal transcription factor and potential regulator is not unprecedented; TBP-related factors regulate transcription in metazoans (Goodrich and Tjian, 2010; Duttke et al., 2014; Wang et al., 2014), and multiple TBP and TFB paralogs regulate transcription in a gene-specific fashion in archaea (Facciotti et alo, 2007).

\section{Evolution of TFE $\beta$ and hRPC39-like proteins in archaea and eukaryotes}

The archaeal TFE $\alpha / \beta$ factor provides us with a missing link in the evolutionary history of the archaeal and multiple eukaryotic transcription machineries (Figure 8B). In the most parsimonious scenario, the archaeal 'parent' of eukaryotes (Yutin et al., 2008; Guy and Ettema, 2011) included a TFE $\alpha / \beta$ factor with the domain architecture we have discovered and described here. After the split of the archaeal and eukaryotic lineages about 2 billion years ago, and following gene duplication and speciation of both TFE $\alpha$ and $\beta$ subunits in eukaryotes, the FeS domain of TFIIE $\beta$ was lost in the RNAPII system along with the evolution of a new dimer interface (Grünberg et al., 2012). The loss of the FeS cluster in TFIIE $\beta$ might have evolved via a route analogous to the apparent loss of the FeS cluster in yeast C34 (Figure 2G).

In the RNAPIII system the hRPC62/39 complex with the addition of a third, not universally conserved subunit hRPC32 (C31 in yeast) (Proshkina et al., 2006) became stably incorporated into RNAPIII, most likely via a WH domain duplication turning the single WH domain in TFE $\alpha$ to four WH domains of hRPC62 (Lefèvre et al., 2011) which provided a drastically enhanced binding surface (Vannini et al., 2010). This may have provided a selective advantage considering that transcription by RNAPIII is characterised by high rates of transcription reinitiation and short transcript length. It seems likely that the essential role of the FeS cluster in TFE $\alpha / \beta$ dimerization is conserved in hRPC62/39 since a truncated form of human hRPC39 encompassing the third WH domain and the FeS domain supports dimerization (Lefèvre et al., 2011).

Overall the diverse activities of TFE $\alpha / \beta$ described here are consistent with the activities of TFE $\alpha$ from euryarchaeal transcription systems that do not encompass TFE $\beta$ homologues such as $M$. jannaschii and Pyrococcus furiosus (Hanzelka et al., 2001; Werner and Weinzierl, 2005; Naji et al., 2007; Kostrewa et al., 2009; Grohmann et al., 2011). Monomeric Sso TFE $\alpha$ did not stimulate PIC formation or transcription (Figures 6B, 7C). It is worth noting that Sso TFE $\alpha$ binds to the RNAP clamp (Figure 5) and in vitro transcription experiments suggest that Sso TFE $\alpha$ competes with Sso TFE $\alpha / \beta$ for incorporation into the PIC (data not shown). Hence, Sso TFE $\alpha$ appears to be recruited to the PIC but does not exert any detectable effect on transcriptional activity. Consistent with our results, a previous study suggested that monomeric Sso TFE $\alpha$ does not affect transcription output from the viral T6 promoter when non-limiting TBP concentrations similar to those used in this study were used (Bell et al., 2001).

Loss of the TFE $\beta$ encoding gene in euryarchaeal species might be compensated for by changes in TFE $\alpha$ and RNAP, particularly regarding the mechanisms and dynamics of clamp opening. The structure of the Thermococcus kodakaraensis RNAP revealed the clamp in an open conformation (Jun et al., 2014) and this species has no TFE $\beta$ homologue. The Sso RNAP and yeast RNAPII crystallise with a closed clamp, and both systems utilise TF(II)E $\beta$ for initiation (Bushnell and Kornberg, 2003; Hirata et al., 2008; Korkhin et al., 2009; this work). We envisage a model where TFE engages with and possibly opens the RNAP clamp, which stimulates open complex formation and transcription. In organisms that lack TFE $\beta$ the opening of the clamp occurs more readily, which is reflected in the corresponding RNAP structures; in some species clamp opening occurs entirely factor-indpendent since genes encoding TFE $\alpha$ and TFE $\beta$ homologues are missing in the euryarchaeal Thermoplasmata class. What was the selective advantage that led to the emergence of TFE $\beta$ ? In budding yeast, the Tfa1 WH domain 1 interacts with the Ssl2 subunit (termed XPB in human and archaea) of TFIIH (Grünberg et al., 2012) which facilitates DNA nucleotide excision repair (Rouillon and White, 2011) and ATPdependent DNA melting during transcription initiation (Holstege et alo, 1996; Kim et alo, 2000). In archaea, open complex formation occurs spontaneously due to the torsionally strained topology of the promoter DNA in the PIC (Nagy et al., 2015) and there is no evidence of any involvement of archaeal 
$\mathrm{XPB}$ in transcription initiation. However, the TFE $\beta$ WH domain would be poised to enable the recruitment or integration of XPB-containing complexes into the PIC.

The discovery and characterisation of Sso TFE $\alpha / \beta$ provides an important piece of the evolutionary puzzle of TFIIE-like proteins. It suggests that streamlining occurred in the evolution of the archaeal transcription machinery, that is, the loss of $t f e \beta$ in several species (Blombach et alo, 2009). In eukaryotes on the other hand, evolution led to an increase in complexity chiefly by WH domain duplication events.

\section{Materials and methods}

\section{Molecular cloning}

Cloning via restriction sites was performed with PCR products amplified from $S$. solfataricus str. P2 genomic DNA. For co-expression of Sso TFE $\alpha$ (Sso0266) and Sso TFE $\beta$-His (Sso 0944) a bicistronic expression construct was produced ( $\mathrm{p} 1076)$. A corresponding construct for co-expression of hRPC62 and hRPC39 (p1159) was created likewise. To generate a pRSF-1b-based vector for expression of Cterminally His-tagged Sso TFE $\beta$, the gene was amplified from a pET21a+ expression vector including the vector-encoded His-tag and inserted into the pRSF-1b vector that does not encode a His-Tag otherwise. The resulting construct ( $\mathrm{p} 1077)$ was also used as backbone to generate the different Sso TFE $\beta$ truncation mutants. All plasmids generated by restriction enzyme-based cloning are listed in Supplementary file 1. Site-directed mutagenesis of TFE $\alpha$ and TFE $\beta$ was performed to yield single or double amino acid substitutions as listed in Supplementary file 2. For the deletion of nucleotides coding for residues 46-52 in Sso TFE $\alpha$, a 5'-end phosporylated non-overlapping primer pair was used according to the Phusion Site-directed mutagenesis protocol (Thermo Scientific/Fisher, Loughborough, United Kingdom). All oligonucleotide sequences are listed in Supplementary file 3. The sequence of all constructs was verified.

\section{Recombinant protein expression and purification}

For isolation of Sso RNAP, pSVA158 was transformed into S. sulfolobus M16 cells resulting in the expression of a C-terminally His 10 -tagged RNAP subunit Rpo8 and homologous expression was carried out as described previously (Albers et al., 2006). Cells were harvested by centrifugation, snap frozen in liquid $\mathrm{N}_{2}$ and stored at $-80^{\circ} \mathrm{C}$. Cells were resuspended in $30 \mathrm{ml} \mathrm{N}$ buffer $(25 \mathrm{mM} \mathrm{Tris} / \mathrm{HCl} \mathrm{pH}$ 8.0, $10 \mathrm{mM} \mathrm{MgCl}_{2}, 100 \mu \mathrm{M} \mathrm{ZnSO}_{4}, 5 \mathrm{mM}$ 2-mercapto-ethanol, $10 \%$ glycerol) with $100 \mathrm{mM} \mathrm{NaCl}$ $(\mathrm{N}(100)$, salt concentration given in parenthesis) supplemented with EDTA-free protease inhibitor tablets (Roche, Burgess Hill, United Kingdom) and DNase I (Sigma, Gillingham, United Kingdom). After disruption using a French Pressure cell at 16,000 psi the lysate was cleared by centrifugation and filtration. The salt concentration was adjusted to $500 \mathrm{mM} \mathrm{NaCl}$ and the lysate was loaded onto a $1 \mathrm{ml}$ Histrap ff cartridge (GE Life Sciences, Uppsala, Sweden). The column was washed with $10 \mathrm{ml} \mathrm{N}(500)$, $20 \mathrm{mM}$ imidazole and RNAP was eluted using a gradient to $250 \mathrm{mM}$ imidazole. Elution fractions were combined, diluted with $\mathrm{N}(0)$ buffer to $100 \mathrm{mM} \mathrm{NaCl}$, and loaded onto a $1 \mathrm{ml}$ HiTrap Heparin HP cartridge (GE Life Sciences). Sso RNAP was eluted with a $10 \mathrm{ml}$ gradient to $N(1000)$ yielding a single sharp peak for Sso RNAP. The peak fractions were combined and desalted using a PD-10 desalting column (GE Life Sciences) to N(150).

All heterologous expression was performed in E. coli Rosetta 2(DE3) (Merck Millipore, Billerica, MA) or BL21 Star (DE3) (Life Technologies, Paisley, United Kingdom) in enriched growth medium at $37^{\circ} \mathrm{C}$ according to standard procedures. For the expression of Sso TFE $\beta$, Sso TFE $\alpha / \beta$ and all the mutant versions of these proteins as well as hC62/39 in BL21 Star (DE3) $0.5 \mathrm{mM}$ L-cysteine and $0.5 \mathrm{mM}$ Ammonium ferric citrate were added after induction in order to maximize FeS cluster occupancy and expression was allowed for $2.5 \mathrm{hr}$. Sso TBP and Sso TFB-His were expressed from plasmids p1121, 1087, and p1168 respectively and purified as previously described (Gietl et al., 2014). Sso TFE $\alpha$ was expressed in Rosetta 2(DE3) cells from plasmid p988. Cells were resuspended in $N(300)$ and disrupted using a using a French Pressure cell at 16,000 psi. The lysate was cleared by centrifugation, incubated for $30 \mathrm{~min}$ at $70^{\circ} \mathrm{C}$ and denatured host proteins were removed by centrifugation. The heat-stable lysate was further purified on a HiPrep 16/60 Sephacryl S-100 HR column (GE Life Sciences). TFE $\alpha$ containing fractions were combined and threefold diluted with $\mathrm{N}(0)$ to $100 \mathrm{mM} \mathrm{NaCl}$. The protein was loaded onto a UnoQ-1 column (Bio-Rad, Hemel Hempstead, United Kingdom) and eluted using a $10 \mathrm{ml}$ gradient to $\mathrm{N}(1000)$. The purity of TFE $\alpha$ was judged by SDS-PAGE and fractions containing 
TFE $\alpha$ with $>95 \%$ purity were combined and concentrated by ultrafiltration, snap frozen in liquid nitrogen and stored at $-80^{\circ} \mathrm{C}$. For the purification of Sso TFE $\alpha / \beta$-His and all mutant versions, cells were resuspended in TK buffer $\left(20 \mathrm{mM}\right.$ Tris/ $\mathrm{HCl} \mathrm{pH} 8.0,100 \mu \mathrm{M} \mathrm{ZnSO}_{4}, 5 \mathrm{mM}$ DTT) with $500 \mathrm{mM} \mathrm{KCl}$ (TK(500), salt concentration given in parenthesis) supplemented with $5 \mathrm{mM}$ imidazole and disrupted by sonication. After centrifugation the supernatant was incubated at $65^{\circ} \mathrm{C}$ for 20 min and centrifuged again. The heat-stable supernatant was purified on a $1 \mathrm{ml}$ Histrap ff cartridge (GE Life Sciences). Sso $\mathrm{TFE} \alpha / \beta$-His-containing elution fractions were pooled, diluted fivefold with TK(0) buffer to $100 \mathrm{mM} \mathrm{KCl}$ and loaded on a $1 \mathrm{ml} \mathrm{HiTrap} \mathrm{Heparin} \mathrm{HP} \mathrm{cartridge} \mathrm{(GE} \mathrm{Life} \mathrm{Sciences)} \mathrm{and} \mathrm{eluted} \mathrm{with} \mathrm{a} 10 \mathrm{ml}$ gradient to TK(1000). Fractions were concentrated in AMICON ULTRA $0.5 \mathrm{ml}$ MWCO10000 ultrafiltration devices. Aliquots were snap-frozen in liquid nitrogen and stored at $-80^{\circ} \mathrm{C}$ before usage. The whole purification was carried out in a single day to limit oxidation of the Fe-S cluster. For the purification of mutant versions of Sso TFE $\alpha / \beta$-His and WT TFE $\alpha / \beta$-His serving as control the heparin-affinity chromatography was omitted and instead the protein was buffer changed on a PD-10 column (GE Life Sciences) to TK(150). Human N-terminally His-tagged C62/C39 was purified as follows: Cells were resuspended in TK(150), $20 \mathrm{mM}$ imidazole and disrupted by sonication. After removal of cell debris by centrifugation the supernatant was purified on a $1 \mathrm{ml}$ Histrap $\mathrm{ff}$ cartridge (GE Life Sciences). Elution fractions containing protein were combined and diluted with 0.5 vol water to $100 \mathrm{mM}$ salt. The protein was further purified and buffer exchanged on a $1 \mathrm{ml}$ HiTrap Heparin HP cartridge (GE Life Sciences) equilibrated in $0.1 \mathrm{M} \mathrm{NH}_{4}$-Acetate and eluted as a single peak using a $10 \mathrm{ml}$ gradient to $1 \mathrm{M} \mathrm{NH}_{4}$-Acetate in order to have a protein preparation compatible with native MS. A construct for the expression of a recombinant Sso RNAP clamp module ( $p 1172$ ) was designed according to a similar construct for $P$. furiosus (Martinez-Rucobo et alo, 2011). The Sso RNAP clamp module was purified as described above for TFE $\alpha / \beta$-His. Protein concentrations were determined using the Qubit assay (Life Technologies).

\section{Western blotting and immunodetection}

Polyclonal rabbit antisera against recombinant Sso TBP, Sso TFE $\alpha$ and Sso TFE $\beta$ were raised at Davids Biotechnology (Regensburg, Germany). For the detection of Sso TFB we used antisera raised against S. acidocaldarius TFB (Gietl et al., 2014). Rabbit antiserum against S. solfataricus RpoB was obtained from Steve Bell (Indiana University, USA) (Qureshi et al., 1997). For immunodetection, proteins were generally resolved by $14 \%$ SDS-PAGE, transferred to nitrocellulose membranes using a tank or semidry blotting system, and immunodetection was performed using PBS buffer with 5\% milk powder as blocking reagent. The blots were incubated with the respective rabbit antisera and Dylight 680 conjugated goat anti-rabbit IgG (Thermo Scientific) as secondary antibody, scanned on a Typhoon FLA 9500 scanner (GE Life Sciences) equipped with a $685 \mathrm{~nm}$ laser. For multiplex immunodetection including Alba as loading control, sheep anti-Alba antiserum (obtained from Malcolm White, University of St. Andrews, UK) and donkey Dylight 488 conjugated anti-goat lgG were used alongside Dylight 680 conjugated donkey anti-rabbit IgG (Bethyl Laboratories, Cambridge, United Kingdom). We have previously shown that Alba is stably expressed throughout all growth phases (Blombach et al., 2014).

\section{Homologous expression and purification of Sso0944}

For homologous expression of Sso0944 the gene was cloned into vector pMJ0503 (Jonuscheit et al., 2003). The resulting plasmid p1056 was transformed into S. solfataricus M16 cells and expression was carried out as described previously (Jonuscheit et al., 2003). Cells were resuspended in $20 \mathrm{ml} \mathrm{TK}(150)$ supplemented with $2.5 \mathrm{mM} \mathrm{MgCl}_{2}$, DNase I (Sigma), and EDTAfree protease inhibitor (Roche). Cells were disrupted by threefold passage through a French pressure cell (Thermo Scientific) at 16,000 psi. Cell debris was removed by centrifugation at $30,000 \times g$ and filtration through a $0.22 \mu \mathrm{m}$ filter. The cleared cell lysate was loaded onto a $1 \mathrm{ml} \mathrm{Histrap}$ ff cartridge (GE Life Sciences) equilibrated in TK(150) buffer and the column was washed with $5 \mathrm{ml}$ TK (150) and $5 \mathrm{ml} \mathrm{TK(150)} \mathrm{containing} 20 \mathrm{mM}$ imidazole. Protein was eluted with a $5 \mathrm{ml}$ gradient to 250 $\mathrm{mM}$ imidazole and $0.5 \mathrm{ml}$ fractions were collected. For the more stringent purification presented in Figure 1-figure supplement 1 the second wash step was altered to $20 \mathrm{ml}$ buffer containing $50 \mathrm{mM}$ imidazole. 


\section{Cell lysate fractionation}

S. solfataricus P2 cells were grown in Brock medium, $0.1 \%$ NZ-amine, $0.2 \%$ sucrose to late exponential growth phase $\left(\mathrm{OD}_{600}=1.1\right)$, harvested by centrifugation and stored at $-80^{\circ} \mathrm{C} .5 \mathrm{~g}$ cells were resuspended in $20 \mathrm{ml} \mathrm{TK}(150), 10 \mathrm{mM} \mathrm{MgCl}_{2}$, supplemented with EDTA-free protease inhibitor (Roche) and $150 \mu$ DNase I (Sigma) and passed thrice through a French Pressure cell at 16,000 psi. The lysate was cleared by centrifugation $\left(45,000 \times g, 1 \mathrm{hr}\right.$ at $\left.4^{\circ} \mathrm{C}\right)$ and passage through a $0.22 \mu \mathrm{m}$ filter. $250 \mu \mathrm{l}$ of lysate $(10 \mathrm{mg} / \mathrm{ml}$ protein content) were fractionated on a Superose $1210 / 300 \mathrm{GL}$ column (GE Life Sciences) with $0.5 \mathrm{ml}$ fraction size. Fractions were analysed by immunodetection.

\section{Quantitative immunodetection}

S. solfataricus P2 cells were grown in shake flasks in 1 litre Brock medium (Zaparty et al., 2010) supplemented with $0.1 \%$ (wt/vol) tryptone and $0.2 \%$ glucose at $76^{\circ} \mathrm{C}$ under aerobic conditions in triplicate. After $24 \mathrm{hr}$ the cultures reach O.D.600 $=0.4$ (exponential growth phase) and $50 \mathrm{ml}$ were withdrawn. After $96 \mathrm{hr}$ at O.D.600 $=2.3$ (stationary growth phase) $10 \mathrm{ml}$ were withdrawn. All samples were immediately chilled on ice, cells were harvested by centrifugation and stored at $-80^{\circ} \mathrm{C}$. Cells were then resuspended in $1 \mathrm{ml}$ TK(150) buffer supplemented with $2.5 \mathrm{mM} \mathrm{MgCl}_{2}$, DNase I (Sigma), and EDTA-free protease inhibitor (Roche). Cells were disrupted using a cup sonicator and lysates were cleared by centrifugation $\left(20 \mathrm{~min}\right.$ at $20,000 \times \mathrm{g}, 4^{\circ} \mathrm{C}$ ). Protein concentrations in the cleared lysates were determined using the Qubit assay (Life Technologies). Lysates were diluted to $1.2 \mathrm{mg} / \mathrm{ml}$, mixed with SDS loading dye and resolved by $14 \%$ SDS-PAGE. Proteins were transferred onto nitrocellulose membranes by semi-dry blotting. To ensure equal loading and transfer, the membranes were stained with Ponceau S before immunodetection performed as described above. In parallel, a twofold dilution series of recombinant untagged TFE $\alpha / \beta$ or TBP was loaded on to the gels to generate a calibration curve used to allow determination of the expression levels.

\section{Saci_1342 disruption attempts}

The procedure to disrupt Saci_1342 basically followed the method described by (Wagner et al., 2012) using pyrEF as marker gene and the uracil-auxotrophic S. acidocaldarius strain MW001 as parental strain. The upstream and downstream flanks of Saci_1342 (751 bp upstream flank including the initial 9 bp of Saci_1342 and 700 bp downstream flank including 26 bp at the 3' end of Saci_1342, as this region overlaps with the convergent ORF Saci_1343) were PCR-amplified with primer pairs FW282/283 and FW284/FW285, respectively, fused by overlap extension PCR and inserted into vector pSVA406 via $\mathrm{Ncol}$ and BamHI restriction sites yielding plasmid p1058. After EsaBC41 cytosine-methylation in E. coli (Grogan, 2003) p1058 was transformed into electrocompetent MW001 cells and single-crossover integrants were selected on uracil-deficient plates. Four single-crossover integrants were selected and plated on uracil and 5-FOA containing plates for counterselection against pyrEF triggering excision of p1058. Strain MW001 Saci1162::Saci1342 was constructed plasmid 1112 similar to (Meyer and Albers, 2014). Strain MW001 Saci1162::Saci_1342 was then transformed with p1058 as described above. Successful replacement of Saci_1162 and deletion of Saci_1342 was confirmed by DNA sequencing.

\section{Co-purification assays}

For co-purification assays, pET-21a(+) and pRSF-1b-based vectors for the expression of Sso TFE $\alpha$ and Sso TFE $\beta$ or their mutant versions were co-transformed into BL21* (DE3) cells (Life Technologies). Proteins were expressed in enriched growth medium for $2.5 \mathrm{hr}$ at $37^{\circ} \mathrm{C}$ after induction with IPTG. Cells from $400 \mathrm{ml}$ culture were resuspended in $4 \mathrm{ml} \mathrm{TK}(500)$ buffer and disrupted by sonication. The cleared lysate was incubated at $65^{\circ} \mathrm{C}$ for $20 \mathrm{~min}$. The heat-stable supernatant was mixed with $0.2 \mathrm{ml}$ pre-equilibrated His-Select resin (Sigma-Aldrich) and incubated for $10 \mathrm{~min}$ at $4^{\circ} \mathrm{C}$. The resin was washed with $5 \mathrm{ml}$ buffer TK(500) containing $5 \mathrm{mM}$ imidazole. Bound proteins were eluted by addition of $1 \mathrm{ml}$ TK (500) containing $250 \mathrm{mM}$ imidazole. Samples were analysed by Tris-Tricine SDS-PAGE.

\section{Binding assays with the RNAP clamp}

$10 \mu \mathrm{M}$ Clamp module were mixed with $10 \mu \mathrm{M}$ of SsoTFE $\alpha \beta$ or mutants thereof in TK(250) buffer, $10 \%$ glycerol, $10 \mathrm{mM} \mathrm{MgCl} 2$ and incubated at $37^{\circ} \mathrm{C}$ for $10 \mathrm{~min}$. After brief centrifugation, $250 \mu \mathrm{l}$ were loaded on a Superose 12 10/300 GL column (GE Life Sciences) equilibrated in the same buffer plus 
$0.05 \%$ TWEEN 20 . The fraction size was $0.5 \mathrm{ml}$. The elution profile of the respective proteins was visualized on silver-stained SDS-PAGE gels.

\section{UV-vis spectroscopy}

All samples were measured in an AvaSpec-2048 fiber optic spectrophotometer using a $10 \mathrm{~mm}$ pathlength quartz cuvette at a protein concentration of $5 \mu \mathrm{M}$ and $2 \mu \mathrm{M}$ for Sso TFE $\alpha / \beta$ and C62/39, respectively.

\section{CWEPR}

For EPR measurements Sso TFE $\alpha / \beta$-His and human N-terminally His-tagged C62/C39 samples were aerobically purified and concentrated to 550 and $86 \mu \mathrm{M}$ respectively, based on $\mathrm{A} 280$ absorption and the molar extinction coefficient. As-isolated samples were transferred directly to EPR quartz tubes and flash frozen in liquid nitrogen. Reduced samples were prepared by supplementing a fraction of the same protein stock with $20 \mathrm{mM}$ sodium dithionite by adding 1/100 vol of a $2 \mathrm{M}$ solution to the buffer before freezing. All samples were stored in liquid nitrogen prior to cW-EPR experiments, and showed no change in EPR signal over a period of several months. Cw-EPR measurements were performed on a Bruker EMXplus spectrometer operating at $9.4 \mathrm{GHz}$ (X-band) equipped with a 4122SHQE resonator, with an Oxford Instruments ESR900 cryostat for measurements at cryogenic temperatures. Typically spectra were acquired in the temperature interval $10-40 \mathrm{~K}$ to enable FeS cluster identification. Measurements were performed with a magnetic field sweep from 50 to $600 \mathrm{mT}$ (to allow the detection of possible high and low spin Fe species), a microwave power of $2 \mathrm{~mW}$, modulation amplitude of $0.5 \mathrm{mT}$ and a modulation frequency of $100 \mathrm{kHz}$. Three independent preparations were tested, all of which gave consistent results. Spin quantification was carried out by comparison with a standard solution of $\mathrm{Cu}($ II)EDTA according to the method reported in (chasteen). The magnetic field was calibrated with a bismuth doped silicon sample.

\section{nESI mass spectrometry}

For nano-electrospray ionization mass spectrometry of TFE $\alpha / \beta$-His the heparin-affinity chromatography step was carried out with $0.3-1 \mathrm{M} \mathrm{NH}_{4}$-Acetate. Prior to analysis by native mass spectrometry, protein samples were buffer-exchanged into $150 \mathrm{mM}$ ammonium acetate and concentrated to $\sim 10 \mu \mathrm{M}$ using Amicon Ultra 0.5-ml centrifugal filters (Millipore). Mass spectrometry experiments were carried out on a first-generation Synapt HDMS (Waters, Manchester, UK) Quadrupole-TOF, traveling wave ion mobility mass spectrometer (Pringle et al., 2007). Samples (2- to 3- $\mu$ l aliquots) were introduced to the mass spectrometer by means of nanoelectrospray ( $\mathrm{nESI}$ ) ionization using gold-coated capillaries that were prepared in-house. Typical instrumental parameters were as follows: source pressure, 5 mbar; capillary voltage, 1.0-1.3 kV; cone voltage, $40 \mathrm{~V}$; trap energy, $15 \mathrm{~V}$; transfer energy, $10 \mathrm{~V}$; bias, $2.0 \mathrm{~V}$. For tandem MS experiments, the backing pressure was reduced to $1.4 \mathrm{mbar}$ and the trap and transfer voltages were increased to $60 \mathrm{~V}$ and $20 \mathrm{~V}$ respectively. MS were smoothed and peak-centered in MassLynx v4.1 (Waters, Elstree, United Kingdom).

\section{EMSA and potassium permanganate footprinting}

$15 \mu$ samples contained $10 \mathrm{mM}$ MOPS pH 6.5, $11 \mathrm{mM} \mathrm{MgCl}, 150 \mathrm{mM}$ salt $(115 \mathrm{mM} \mathrm{KCl}, 27 \mathrm{mM} \mathrm{NaCl}$, $8 \mathrm{mM}$ K-Acetate), 10\% glycerol, $5 \mu \mathrm{g} / \mathrm{ml}$ heparin, $63 \mathrm{nM}$ TFB, $250 \mathrm{nM}$ TBP, 270 ng RNAP (45 nM) and $125 \mathrm{fmol}$ of ${ }^{32} \mathrm{P} 5^{\prime}$-labelled dsDNA templates. Samples were incubated for $5 \mathrm{~min}$ at $65^{\circ} \mathrm{C}$ before loading onto a $5 \%$ native Tris-Glycine gels $(2.5 \%$ glycerol, $1 \mathrm{mM}$ DTT). To generate antibodysupershifts, the Protein A-purified anti-TFE $\alpha$, anti-TFE $\beta$, or anti-TBP $(0.7 \mu \mathrm{g})$ antiserum was added to the incubated samples and incubation continued for $15 \mathrm{~min}$ at room temperature prior to gel loading. Gels were run at $150 \mathrm{~V}$, dried and radiolabelled DNA was detected by phosphorimagery.

For potassium permanganate footprinting the samples were as described above but scaled up to $23 \mu \mathrm{l}$. TBP and TFB concentrations were raised to $1 \mu \mathrm{M}$ and $125 \mathrm{nM}$, respectively, $\mathrm{MgCl}_{2}$ to $26 \mathrm{mM}$, and DTT was reduced to $0.5 \mathrm{mM}$. Samples were incubated for $5 \mathrm{~min}$ at $65^{\circ} \mathrm{C}$ before the addition of $2 \mu \mathrm{l}$ $50 \mathrm{mM} \mathrm{KMnO}_{4}$ (4 mM final concentration). Samples were further incubated at $65^{\circ} \mathrm{C}$ for 5 min before the addition of $1.5 \mu \mathrm{l} 2$-mercaptoethanol to stop the reaction. After Proteinase K-treatment and ethanolprecipitation, samples were resuspended in $50 \mu 1 \mathrm{M}$ piperidine and incubated at $90^{\circ} \mathrm{C}$ for $30 \mathrm{~min}$. After chloroform-extraction, samples were ethanol-precipitated and resuspended in formamide loading dye. 
Samples were resolved on a $10 \%$ polyacrylamide, $7 \mathrm{M}$ Urea, $1 \times$ TBE sequencing gels. Radiolabelled DNA was detected by phosphor imagery.

\section{Abortive and productive transcription assays}

$15 \mu \mathrm{l}$ samples for abortive transcription assays contained $500 \mathrm{fmol}$ of dsDNA template pol592/593 (homoduplex) or pol592/603 (-4 to -1 heteroduplex), $250 \mu \mathrm{M}$ ApG dinucleotide, $50 \mu \mathrm{M}$ ATP (containing $\left[\alpha-{ }^{32}\right.$ P]-ATP), $125 \mathrm{nM}$ TFB, $1 \mu \mathrm{M}$ TBP, $270 \mathrm{ng}$ RNAP $(45 \mathrm{nM})$, and $1 \mu \mathrm{M}$ TFE $\alpha / \beta$. Salt, buffer and heparin concentrations were identical to those used for the mobility shift experiments. Samples were incubated for $10 \mathrm{~min}$ at $65^{\circ} \mathrm{C}$ before addition of 1 vol formamide loading dye. $5 \mu$ of the samples was loaded on a $20 \% 7 \mathrm{M}$ Urea, $1 \times$ TBE PAGE mini-gel and resolved at $300 \mathrm{~V}$ for $45 \mathrm{~min}$. Signals were detected by phosphorimagery and quantified using the ImageQuant TL software package (GE Life Sciences). The quantification of TFE $\alpha / \beta$ stimulation was based on three technical replicates. The signal obtained in absence of TFB was subtracted as background.

For promoter-directed in vitro transcription, different promoters fused to a C-less cassette derived from a synthetic $390 \mathrm{nt}$ G-less cassette (kindly provided by James Goodrich, CO) (Sawadogo and Roeder, 1985) were cloned into PGEM-T (Promega) (Supplementary file 4). All plasmids were treated converted to relaxed topology with E. coli Topoisomerase I (NEB) according to manufacturer's protocol. Transcription reactions were modified as follows: samples contained $500 \mu \mathrm{M}$ ATP/GTP, $2.5 \mu \mathrm{M}$ UTP (containing [ $\alpha^{-32}$ P]-UTP) and $200 \mathrm{ng}$ of the respective plasmid template. Reactions were stopped after $10 \mathrm{~min}$ by the addition of 9 vol stop mix $(0.3 \mathrm{M}$ Na-acetate pH 5.2, $10 \mathrm{mM}$ EDTA, 0.5\% SDS, $100 \mu \mathrm{g} / \mathrm{ml}$ glycogen and trace amounts of radiolabelled oligonucleotide serving as recovery marker). Samples were purified by phenol/ chloroform extraction and ethanol-precipitation and resuspended in formamide-loading dye. Sample were resolved on a $10 \%$ polyacrylamide, $7 \mathrm{M}$ Urea, $1 \times$ TBE sequencing gel. Transcripts were detected by phosphor imagery and quantification of bands was performed using the ImageQuant TL software (GE Life Sciences). The signals were normalized using the recovery marker. When activities on different promoters were compared, the different specific activities of the transcripts were taken into account.

\section{Acknowledgements}

The authors wish to thank Alessandro Vannini from the Institute for Cancer Research for the gift of the human hRPC62/39 expression vector, Peter Rich (UCL ISMB) for help with UV-Vis spectroscopy, and Richard Cammack for stimulating discussions of cubane FeS cluster EPR spectra. This work was supported by a Wellcome Trust investigator award to FW (079351/Z/06/Z), and FB was supported by a research fellowship from the German Research Foundation (DFG BL 1189/1-1). JR and SVA were supported by intramural fund of the Max Planck Society. JY was supported by a Wellcome Trust PhD studentship.

\section{Additional information}

Funding

\begin{tabular}{|c|c|c|}
\hline Funder & Grant reference & Author \\
\hline Wellcome Trust & 079351/Z/06/Z & Finn Werner \\
\hline $\begin{array}{l}\text { Deutsche } \\
\text { Forschungsgemeinschaft }\end{array}$ & $\begin{array}{l}\text { Research Fellowship DFG } \\
\text { BL 1189/1-1 }\end{array}$ & Fabian Blombach \\
\hline Max-Planck-Gesellschaft & intramural fund & $\begin{array}{l}\text { Julia Reimann, Sonja V } \\
\text { Albers }\end{array}$ \\
\hline Wellcome Trust & PhD fellowship & Jun Yan \\
\hline $\begin{array}{l}\text { Biotechnology and } \\
\text { Biological Sciences } \\
\text { Research Council (BBSRC) }\end{array}$ & BB/H019332/1 & Finn Werner \\
\hline
\end{tabular}

The funders had no role in study design, data collection and interpretation, or the decision to submit the work for publication. 
Author contributions

FB, KT, Conception and design, Acquisition of data, Analysis and interpretation of data, Drafting or revising the article; ES, Acquisition of data, Analysis and interpretation of data, Drafting or revising the article; TF, Conception and design, Acquisition of data, Analysis and interpretation of data; JY, Acquisition of data, Analysis and interpretation of data; JR, Conception and design, Acquisition of data; CS, KLS, Conception and design, Analysis and interpretation of data; SVA, Conception and design, Contributed unpublished essential data or reagents; CWMK, FW, Conception and design, Analysis and interpretation of data, Drafting or revising the article

Author ORCIDs

Christopher WM Kay, (iD http://orcid.org/0000-0002-5200-6004

\section{Additional files}

Supplementary files

- Supplementary file 1. List of plasmids generated by restriction enzyme-based cloning. DOI: 10.7554/eLife.08378.022

- Supplementary file 2. List of plasmids generated by PCR-based Site-directed mutagenesis. DOI: 10.7554/eLife.08378.023

- Supplementary file 3. List of oligonucleotides used in this study. DOI: $10.7554 /$ LLife.08378.024

- Supplementary file 4. List of in vitro transcription templates with promoters fused to a C-less cassette.

DOI: $10.7554 /$ LLife.08378.025

\section{References}

Albers SV, Jonuscheit M, Dinkelaker S, Urich T, Kletzin A, Tampe R, Driessen AJ, Schleper C. 2006. Production of recombinant and tagged proteins in the hyperthermophilic archaeon Sulfolobus solfataricus. Applied and Environmental Microbiology 72:102-111. doi: 10.1128/AEM.72.1.102-111.2006.

Aravind L, Anantharaman V, Balaji S, Babu MM, lyer LM. 2005. The many faces of the helix-turn-helix domain: transcription regulation and beyond. FEMS Microbiology Reviews 29:231-262. doi: 10.1016/j.femsre.2004.12.008.

Beinert H, Kennedy MC, Stout CD. 1996. Aconitase as iron-sulfur protein, enzyme, and iron-regulatory protein. Chemical Reviews 96:2335-2374. doi: 10.1021/cr950040z.

Bell SD, Brinkman AB, van der Oost J, Jackson SP. 2001. The archaeal TFIIEalpha homologue facilitates transcription initiation by enhancing TATA-box recognition. EMBO Reports 2:133-138. doi: 10.1093/emboreports/kve021.

Blombach F, Launay H, Snijders AP, Zorraquino V, Wu H, de Koning B, Brouns SJ, Ettema TJ, Camilloni C, Cavalli A, Vendruscolo M, Dickman MJ, Cabrita LD, La Teana A, Benelli D, Londei P, Christodoulou J, van der Oost J. 2014. Archaeal MBF1 binds to $30 \mathrm{~S}$ and $70 \mathrm{~S}$ ribosomes via its helix-turn-helix domain. The Biochemical Journal 462: 373-384. doi: 10.1042/BJ20131474.

Blombach F, Makarova KS, Marrero J, Siebers B, Koonin EV, van der Oost J. 2009. Identification of an ortholog of the eukaryotic RNA polymerase III subunit RPC34 in Crenarchaeota and Thaumarchaeota suggests specialization of RNA polymerases for coding and non-coding RNAs in Archaea. Biology Direct 4:39. doi: 10.1186/ 1745-6150-4-39.

Bushnell DA, Kornberg RD. 2003. Complete, 12-subunit RNA polymerase II at 4.1-A resolution: implications for the initiation of transcription. Proceedings of the National Academy of Sciences of USA 100:6969-6973. doi: 10. 1073/pnas.1130601100.

Carter R, Drouin G. 2010. The increase in the number of subunits in eukaryotic RNA polymerase III relative to RNA polymerase II is due to the permanent recruitment of general transcription factors. Molecular Biology and Evolution 27:1035-1043. doi: 10.1093/molbev/msp316.

Duttke SH, Doolittle RF, Wang YL, Kadonaga JT. 2014. TRF2 and the evolution of the bilateria. Genes \& Development 28:2071-2076. doi: 10.1101/gad.250563.114.

Facciotti MT, Reiss DJ, Pan M, Kaur A, Vuthoori M, Bonneau R, Shannon P, Srivastava A, Donohoe SM, Hood LE, Baliga NS. 2007. General transcription factor specified global gene regulation in archaea. Proceedings of the National Academy of Sciences of USA 104:4630-4635. doi: 10.1073/pnas.0611663104.

Feaver WJ, Henry NL, Bushnell DA, Sayre MH, Brickner JH, Gileadi O, Kornberg RD. 1994. Yeast TFIIE. Cloning, expression, and homology to vertebrate proteins. Journal of Biological Chemistry 269:27549-27553.

Fishburn J, Tomko E, Galburt E, Hahn S. 2015. Double-stranded DNA translocase activity of transcription factor TFIIH and the mechanism of RNA polymerase II open complex formation. Proceedings of the National Academy of Sciences of USA 112:3961-3966. doi: 10.1073/pnas.1417709112. 
Gietl A, Holzmeister P, Blombach F, Schulz S, von Voithenberg LV, Lamb DC, Werner F, Tinnefeld P, Grohmann D. 2014. Eukaryotic and archaeal TBP and TFB/TF(II)B follow different promoter DNA bending pathways. Nucleic Acids Research 42:6219-6231. doi: 10.1093/nar/gku273.

Goodrich JA, Tjian R. 2010. Unexpected roles for core promoter recognition factors in cell-type-specific transcription and gene regulation. Nature Reviews Genetics 11:549-558. doi: 10.1038/nrg2847.

Grogan DW. 2003. Cytosine methylation by the Sual restriction-modification system: implications for genetic fidelity in a hyperthermophilic archaeon. Journal of Bacteriology 185:4657-4661. doi: 10.1128/JB.185.15.46574661.2003.

Grohmann D, Nagy J, Chakraborty A, Klose D, Fielden D, Ebright RH, Michaelis J, Werner F. 2011. The initiation factor TFE and the elongation factor Spt $4 / 5$ compete for the RNAP clamp during transcription initiation and elongation. Molecular Cell 43:263-274. doi: 10.1016/j.molcel.2011.05.030.

Grünberg S, Warfield L, Hahn S. 2012. Architecture of the RNA polymerase II preinitiation complex and mechanism of ATP-dependent promoter opening. Nature Structural \& Molecular Biology 19:788-796. doi: 10.1038/nsmb.2334.

Guy L, Ettema TJ. 2011. The archaeal 'TACK' superphylum and the origin of eukaryotes. Trends in Microbiology 19:580-587. doi: 10.1016/j.tim.2011.09.002.

Guzman E, Lis JT. 1999. Transcription factor TFIIH is required for promoter melting in vivo. Molecular and Cellular Biology 19:5652-5658.

Hanzelka BL, Darcy TJ, Reeve JN. 2001. TFE, an archaeal transcription factor in Methanobacterium thermoautotrophicum related to eucaryal transcription factor TFIIEalpha. Journal of Bacteriology 183:1813-1818. doi: 10.1128/JB.183.5.1813-1818.2001.

Hausner W, Wettach J, Hethke C, Thomm M. 1996. Two transcription factors related with the eucaryal transcription factors TATA-binding protein and transcription factor IIB direct promoter recognition by an archaeal RNA polymerase. The Journal of Biological Chemistry 271:30144-30148. doi: 10.1074/jbc.271.47. 30144.

He Y, Fang J, Taatjes DJ, Nogales E. 2013. Structural visualization of key steps in human transcription initiation. Nature 495:481-486. doi: 10.1038/nature11991.

Hirata A, Klein BJ, Murakami KS. 2008. The X-ray crystal structure of RNA polymerase from Archaea. Nature 451: 851-854. doi: 10.1038/nature06530.

Holstege FC, Tantin D, Carey M, van der Vliet PC, Timmers HT. 1995. The requirement for the basal transcription factor IIE is determined by the helical stability of promoter DNA. EMBO Journal 14:810-819.

Holstege FC, van der Vliet PC, Timmers H. 1996. Opening of an RNA polymerase II promoter occurs in two distinct steps and requires the basal transcription factors IIE and IIH. EMBO Journal 15:1666-1677.

Jonuscheit M, Martusewitsch E, Stedman KM, Schleper C. 2003. A reporter gene system for the hyperthermophilic archaeon Sulfolobus solfataricus based on a selectable and integrative shuttle vector. Molecular Microbiology 48: 1241-1252. doi: 10.1046/j.1365-2958.2003.03509.x.

Jun SH, Hirata A, Kanai T, Santangelo TJ, Imanaka T, Murakami KS. 2014. The X-ray crystal structure of the euryarchaeal RNA polymerase in an open-clamp configuration. Nature Communications 5:5132. doi: 10.1038/ ncomms6132.

Kim TK, Ebright RH, Reinberg D. 2000. Mechanism of ATP-dependent promoter melting by transcription factor IIH. Science 288:1418-1422. doi: 10.1126/science.288.5470.1418.

Korkhin Y, Unligil UM, Littlefield O, Nelson PJ, Stuart DI, Sigler PB, Bell SD, Abrescia NG. 2009. Evolution of complex RNA polymerases: the complete archaeal RNA polymerase structure. PLOS Biology 7:e102. doi: 10. 1371/journal.pbio.1000102.

Kostrewa D, Zeller M, Armache K, Seizl M, Leike K, Thomm M, Cramer P. 2009. RNA polymerase II-TFIIB structure and mechanism of transcription initiation. Nature 462:323-330. doi: 10.1038/nature08548.

Kuldell NH, Buratowski S. 1997. Genetic analysis of the large subunit of yeast transcription factor IIE reveals two regions with distinct functions. Molecular and Cellular Biology 17:5288-5298.

Lefèvre S, Dumay-Odelot H, El-Ayoubi L, Budd A, Legrand P, Pinaud N, Teichmann M, Fribourg S. 2011. Structurefunction analysis of hRPC62 provides insights into RNA polymerase III transcription initiation. Nature Structural \& Molecular Biology 18:352-358. doi: 10.1038/nsmb.1996.

Martinez-Rucobo FW, Sainsbury S, Cheung AC, Cramer P. 2011. Architecture of the RNA polymerase-Spt4/5 complex and basis of universal transcription processivity. The EMBO Journal 30:1302-1310. doi: 10.1038/emboj. 2011.64.

Meinhart A, Blobel J, Cramer P. 2003. An extended winged helix domain in general transcription factor E/IIE alpha. The Journal of Biological Chemistry 278:48267-48274. doi: 10.1074/jbc.M307874200.

Meyer BH, Albers SV. 2014. AglB, catalyzing the oligosaccharyl transferase step of the archaeal N-glycosylation process, is essential in the thermoacidophilic crenarchaeon Sulfolobus acidocaldarius. Microbiologyopen 3: 531-543. doi: 10.1002/mbo3.185.

Nagy J, Grohmann D, Cheung AC, Schulz S, Smollett K, Werner F, Michaelis J. 2015. Complete architecture of the archaeal RNA polymerase open complex from single-molecule FRET and NPS. Nature Communications 6:6161. doi: 10.1038/ncomms7161.

Naji S, Grünberg S, Thomm M. 2007. The RPB7 orthologue E' is required for transcriptional activity of a reconstituted archaeal core enzyme at low temperatures and stimulates open complex formation. The Journal of Biological Chemistry 282:11047-11057. doi: 10.1074/jbc.M611674200.

Netz DJ, Stith CM, Stumpfig M, Kopf G, Vogel D, Genau HM, Stodola JL, Lill R, Burgers PM, Pierik AJ. 2012. Eukaryotic DNA polymerases require an iron-sulfur cluster for the formation of active complexes. Nature Chemical Biology 8:125-132. doi: 10.1038/nchembio.721. 
Parvin JD, Sharp PA. 1993. DNA topology and a minimal set of basal factors for transcription by RNA polymerase II. Cell 73:533-540. doi: 10.1016/0092-8674(93)90140-L.

Peterson MG, Inostroza J, Maxon ME, Flores O, Admon A, Reinberg D, Tjian R. 1991. Structure and functional properties of human general transcription factor IIE. Nature 354:369-373. doi: 10.1038/354369a0.

Pringle SD, Giles K, Wildgoose JL, Williams JP, Slade SE, Thalassinos K, Bateman RH, Bowers MT, Scrivens JH. 2007. An investigation of the mobility separation of some peptide and protein ions using a new hybrid quadrupole/ travelling wave IMS/oa-ToF instrument. Int. J. Mass Spectrom 261:1-12. doi: 10.1016/j.ijms.2006.07.021.

Proshkina GM, Shematorova EK, Proshkin SA, Zaros C, Thuriaux P, Shpakovski GV. 2006. Ancient origin, functional conservation and fast evolution of DNA-dependent RNA polymerase III. Nucleic Acids Research 34:3615-3624. doi: $10.1093 / \mathrm{nar} / \mathrm{gkl} 421$.

Qureshi SA, Bell SD, Jackson SP. 1997. Factor requirements for transcription in the Archaeon Sulfolobus shibatae. The EMBO Journal 16:2927-2936. doi: 10.1093/emboj/16.10.2927.

Rouillon C, White MF. 2011. The evolution and mechanisms of nucleotide excision repair proteins. Research in Microbiology 162:19-26. doi: 10.1016/j.resmic.2010.09.003.

Sawadogo M, Roeder RG. 1985. Factors involved in specific transcription by human RNA polymerase Il: analysis by a rapid and quantitative in vitro assay. Proceedings of the National Academy of Sciences of USA 82:4394-4398. doi: 10.1073/pnas.82.13.4394.

Stettler S, Mariotte S, Riva M, Sentenac A, Thuriaux P. 1992. An essential and specific subunit of RNA polymerase III (C) is encoded by gene RPC34 in Saccharomyces cerevisiae. The Journal of Biological Chemistry 267: 21390-21395.

Vannini A, Cramer P. 2012. Conservation between the RNA polymerase I, II, and III transcription initiation machineries. Molecular Cell 45:439-446. doi: 10.1016/j.molcel.2012.01.023.

Vannini A, Ringel R, Kusser AG, Berninghausen O, Kassavetis GA, Cramer P. 2010. Molecular basis of RNA polymerase III transcription repression by Maf1. Cell 143:59-70. doi: 10.1016/j.cell.2010.09.002.

Wagner M, van Wolferen M, Wagner A, Lassak K, Meyer BH, Reimann J, Albers SV. 2012. Versatile genetic Tool box for the Crenarchaeote Sulfolobus acidocaldarius. Frontiers in Microbiology 3:214. doi: 10.3389/fmicb. 2012.00214.

Wang YL, Duttke SH, Chen K, Johnston J, Kassavetis GA, Zeitlinger J, Kadonaga JT. 2014. TRF2, but not TBP, mediates the transcription of ribosomal protein genes. Genes \& Development 28:1550-1555. doi: 10.1101/gad. 245662.114

Werner F, Grohmann D. 2011. Evolution of multisubunit RNA polymerases in the three domains of life. Nature Reviews Microbiology 9:85-98. doi: 10.1038/nrmicro2507.

Werner F, Weinzierl RO. 2002. A recombinant RNA polymerase II-like enzyme capable of promoter-specific transcription. Molecular Cell 10:635-646. doi: 10.1016/S1097-2765(02)00629-9.

Werner F, Weinzierl RO. 2005. Direct modulation of RNA polymerase core functions by basal transcription factors. Molecular and Cellular Biology 25:8344-8355. doi: 10.1128/MCB.25.18.8344-8355.2005.

White MF, Dillingham MS. 2012. Iron-sulphur clusters in nucleic acid processing enzymes. Current Opinion in Structural Biology 22:94-100. doi: 10.1016/j.sbi.2011.11.004.

Williams TA, Foster PG, Cox CJ, Embley TM. 2013. An archaeal origin of eukaryotes supports only two primary domains of life. Nature 504:231-236. doi: 10.1038/nature12779.

Wu CC, Herzog F, Jennebach S, Lin YC, Pai CY, Aebersold R, Cramer P, Chen HT. 2012. RNA polymerase III subunit architecture and implications for open promoter complex formation. Proceedings of the National Academy of Sciences of USA 109:19232-19237. doi: 10.1073/pnas.1211665109.

Yutin N, Makarova KS, Mekhedov SL, Wolf YI, Koonin EV. 2008. The deep archaeal roots of eukaryotes. Molecular Biology and Evolution 25:1619-1630. doi: 10.1093/molbev/msn108.

Zaparty M, Esser D, Gertig S, Haferkamp P, Kouril T, Manica A, Pham TK, Reimann J, Schreiber K, Sierocinski P, Teichmann D, van Wolferen M, von Jan M, Wieloch P, Albers SV, Driessen AJ, Klenk HP, Schleper C, Schomburg D, van der Oost J, Wright PC, Siebers B. 2010. 'Hot standards' for the thermoacidophilic archaeon Sulfolobus solfataricus. Extremophiles 14:119-142. doi: 10.1007/s00792-009-0280-0. 